\title{
VECTOR FIELDS ON MANIFOLDS ${ }^{1}$
}

\section{EMERY THOMAS}

INTRODUCTION. We discuss in this paper various topics involving continuous vector fields on smooth differentiable manifolds. In each case the underlying idea is the same: we aim to study geometric properties of manifolds by means of algebraic invariants. The prototype for this is the theorem of $\mathrm{H}$. Hopf [27] on vector fields.

TheOREM OF Hopf. A compact manifold $M$ has a vector field without zeros if and only if the Euler characteristic of $M$ vanishes.

Recall that the Euler characteristic of $M, \chi M$, is defined by

$$
\chi M=\sum_{i=0}^{n}(-1)^{i} b_{i},
$$

where $n=\operatorname{dim} M$ and $b_{i}=i$ th Betti number of $M\left(=\operatorname{dim}\right.$ of $\left.H_{i}(M ; Q)\right)$. Thus the geometric property of $M$ having a nonzero vector field is expressed in terms of the algebraic invariant $\chi M$. We will discuss extensions of this idea to vector $k$-fields, fields of $k$-planes, and foliations of manifolds.

All manifolds considered will be connected, smooth and without boundary; all maps will be continuous. For background information on manifolds and vector fields see [30], [34] and [67].

1. The index of a tangent $k$-field. By a tangent $k$-field on a manifold $M$, we will mean $k$ tangent vector fields $X_{1}, \cdots, X_{k}$, which are linearly independent at each point of $M$. If a $k$-field is defined at all but a finite number of points, we will say that it is a $k$-field with finite singularities. In this section we discuss an algebraic invariant, the index, which measures whether or not one can alter a $k$-field so as to remove its singularities.

To define the index we assume that the manifold $M$ has been given a simplicial triangulation so that each point of singularity of the $k$-field lies in the interior of an $m$-simplex, where $m=\operatorname{dim} M$. Let $p$

1 This paper is based on an address given before the meeting of the Society at the University of Victoria on June 18,1966, by invitation of the Committee to Select Hour Speakers for Far-Western Regional meetings; received by the editors February 4, 1969. The author is indebted to the U.S. Air Force Office of Scientific Research; the Mathematics Research Institute, E.T.H. (Zürich); the Miller Institute for Basic Research, and the National Science Foundation for support of the research activity described in the paper. 
be a point of singularity, say in the interior of a simplex $\sigma$. The tangent bundle of $M$ restricted to $\sigma$ is isomorphic to the product bundle $\sigma \times R^{m}$. We now assume that $M$ is an oriented manifold, and the above isomorphism is then taken to be orientation preserving. For each point $q$ in $\sigma-\{p\}$ we can regard $\left(X_{1}(q), \cdots, X_{k}(q)\right)$ as an ordered set of $k$ linearly independent vectors in $R^{m}$-that is, as a point in the Stiefel manifold $V_{m, k}$. Since the $k$-field is defined on the boundary of $\sigma, \dot{\sigma}$, we obtain in this way a map $\dot{\sigma} \rightarrow V_{m, k}$. But $\dot{\sigma}$ is an oriented $(m-1)$-sphere, and so the homotopy class of this map is an element of the homotopy group $\pi_{m-1}\left(V_{m, k}\right)$. This class is defined to be the index of the $k$-field at the point $p$. Finally, we define

$$
\operatorname{Index}\left(X_{1}, \cdots, X_{k}\right)=\sum_{p} \operatorname{Index} \text { at } p,
$$

where the sum is taken over all the singular points of the $k$-field. Thus,

$$
\text { Index }\left(X_{1}, \cdots, X_{k}\right) \in \pi_{m-1}\left(V_{m, k}\right) .
$$

In general the index may change if the orientation of the manifold is reversed. (See, for example, Theorem 5 in this section; also, note \$6.)

The definition of the index is really a special case of the idea of the obstruction to a cross-section of a fiber bundle, developed by Steenrod $[65, \S \S 29-34]$. From this point of view one sees that the index is independent of the choices made in its definition, apart from the choice of orientation.

The geometric significance of the index is given by the following result:

Let $M$ be an m-manifold and let $\left(X_{1}, \cdots, X_{k}\right)$ be a $k$-field on $M$ with finite singularities. Then Index $\left(X_{1}, \cdots, X_{k}\right)=0$ if and only if there is on $M$ a $k$-field without singularities which agrees with $\left(X_{1}, \cdots, X_{k}\right)$ on the $(m-2)$-skeleton of $M$.

For a proof see $[65, \S 34.2]$. This result enables us to split into two parts the problem of whether a manifold admits a $k$-field without singularities.

(1) Does $M$ admit a $k$-field with finite singularities?

(2) If so, how does one compute the index?

In this section we restrict attention to the second of these questions; the first question will be dealt with in \$2. (We simply remark in passing that there is no lack of examples of manifolds admitting $k$-fields with finite singularities; e.g., every $(k-1)$-connected manifold has such a $k$-field.)

We now compute the index of a $k$-field for $k=1,2$. A 1 -field $X$ is 
simply a field of nowhere zero tangent vectors. Since $V_{m, 1}$ has the homotopy type of the sphere $S^{m-1}$ and since $\pi_{m-1} S^{m-1} \approx Z$, we regard Index $(X)$ as an integer. The theorem of Hopf, given in the Introduction, now takes on the more precise form:

Theorem 1 (Hopf). Let $M$ be a compact manifold and let $X$ be a 1-field on $M$ with finite singularities. Then, Index $X=$ Euler characteristic of $M$.

For a modern proof of Hopf's theorem, see [43]; also [30, p. 258].

We now consider 2-fields with finite singularities, on manifolds of dimension $m$; until the end of the section we assume that $m>4$. The index of such a 2 -field is an element of the homotopy group $\pi_{m-1}\left(V_{m, 2}\right)$, a group which depends on the parity of $m$ as shown below (see [50]): For $m \geqq 5$,

$$
\begin{aligned}
\pi_{m-1}\left(V_{m, 2}\right) & =Z_{2} & & \text { if } m \text { odd, } \\
& =Z \oplus Z_{2} & & \text { if } m \text { even. }
\end{aligned}
$$

We begin with the case $m$ odd, and so the index is simply a mod 2 integer. To compute this we consider separately the cases $m \equiv 3 \bmod$ 4 and $m \equiv 1 \bmod 4$.

THEOREM 2. Let $M$ be a compact orientable manifold of dimension $m$, where $m \equiv 3 \bmod 4$ and $m \geqq 7$. If $\left(X_{1}, X_{2}\right)$ is a 2-field on $M$ with finite singularities, then Index $\left(X_{1}, X_{2}\right)=0$ as a mod 2 integer.

The proof is given in [74].

To discuss the case $m \equiv 1 \bmod 4$ we need to introduce additional invariants. First, for any manifold $M$ we have the Stiefel-Whitney classes,

$$
w_{i} M \in H^{i}\left(M ; Z_{2}\right), \quad i \geqq 0
$$

(see [30], [43], [71] for definitions). Recall that a manifold $M$ is orientable if and only if $w_{1} M=0$. Further, an orientable manifold is called a spin manifold if $w_{2} M=0$. (This implies that the structure group of the tangent bundle of $M$ can be taken to be the Spinor group. See [45].) Our result for $m \equiv 1 \bmod 4$ requires that $M$ be a spin manifold. We also will need the mod 2 semicharacteristic, $\hat{\chi}_{2} M$, introduced by Kervaire [31]. Suppose that $\operatorname{dim} M=2 q+1$; we define

$$
\hat{\chi}_{2} M=\sum_{i=0}^{q}\left(\operatorname{dim} H_{i}\left(M ; Z_{2}\right)\right) \bmod 2 .
$$

Thus, $\widehat{\chi}_{2} M$ is a mod 2 integer. 
THEOREM 3. Let $M$ be a compact spin manifold of dimension $m$, where $m \equiv 1 \bmod 4$ and $m \geqq 5$. If $\left(X_{1}, X_{2}\right)$ is any 2 -field on $M$ with finite singularities, then

$$
\operatorname{Index}\left(X_{1}, X_{2}\right)=\hat{\chi}_{2} M
$$

as mod 2 integers.

The proof is given in [76].

Remark (ADDED April 2, 1969). M. Atiyah has informed me of recent research of his on the vector field problem, using the AtiyahSinger Index Theorem [5]. Given a compact, orientable manifold $M$ define the real Kervaire semicharacteristic, $k(M)$, by

$$
k(M)=\left(\sum b_{2 p}\right) \bmod 2,
$$

where $b_{i}$ denotes the $i$ th betti number of $M$. Atiyah proves: Let $M$ be a compact, orientable $(4 k+1)$-manifold, $k>0$, and let $\left(X_{1}, X_{2}\right)$ be a 2-field on $M$ with finite singularities. Then,

$$
\text { Index }\left(X_{1}, X_{2}\right)=k(M) \text {, }
$$

as mod 2 integers.

Note that this result does not require $M$ to be a spin manifold. The relation with Theorem 3 is given by the formula

$$
k(M)-\hat{\chi}_{2} M=w_{2}(M) \cup w_{4 k-1}(M) ;
$$

see a forthcoming paper by Lusztig, Milnor, and Peterson.

We now consider 2 -fields on even dimensional manifolds. As remarked above, the homotopy group $\pi_{m-1}\left(V_{m, 2}\right)$ is isomorphic to $Z \oplus Z_{2}$, when $m$ is even, and so we write the index with two components

$$
\text { Index }\left(X_{1}, X_{2}\right)=\left(Z \text {-Index }\left(X_{1}, X_{2}\right), Z_{2} \text {-Index }\left(X_{1}, X_{2}\right)\right) .
$$

As in the case of Hopf's theorem, one can show that

$$
Z \text {-Index }\left(X_{1}, X_{2}\right)=\chi M,
$$

at least when $M$ is orientable. Our concern here is the calculation of the $Z_{2}$-Index.

THEOREM 4. Let $M$ be a compact orientable $m$-manifold, where $m \equiv 2$ mod 4 and $m \geqq 6$. If $\left(X_{1}, X_{2}\right)$ is any 2-field on $M$ with finite singularities, then

$$
Z_{2} \text {-Index }\left(X_{1}, X_{2}\right)=0
$$

as a mod 2 integer. 
The proof is similar to that of Theorem 2 and is found in [74].

We are left with the case $\operatorname{dim} M \equiv 0 \bmod 4$. For such manifolds (assuming they are compact and oriented) one defines an integral invariant, the signature of $M$, written $\sigma M$. (Following recent usage [5], [33] we call $\sigma M$ the signature rather than the index of $M$, as in [24].) It is easily shown that $\sigma M \equiv \chi M \bmod 2$.

The following result has recently been obtained by D. Frank [13]. (ADDED IN PROOF. M. Atiyah, using the Atiyah-Singer Index Theorem, has obtained this result independently.)

Theorem 5 (Frank-ATiyah). Let $M$ be a compact, oriented manifold of $\operatorname{dim} 4 k, k>1$, and let $\left(X_{1}, X_{2}\right)$ be any 2-field on $M$ with finite singularities. Then,

$$
Z_{2} \text {-Index }\left(X_{1}, X_{2}\right)=\left(\frac{1}{2}\left(\sigma M-(-1)^{k} \chi M\right)\right) \bmod 2 .
$$

The choice of sign is normalized by choosing generators for $\pi_{4 k-1}\left(V_{4 k, 2}\right)$ so that the $Z_{2}$-index $\left(X_{1}, X_{2}\right)=0$, for a 2 -field on $C P^{2 k}$. An earlier result, with more restrictive hypotheses, is given in [76].

Notice that in this theorem, in contrast with Theorems 1-4, the $Z_{2}$-index depends upon the choice of orientation, provided that $\chi M$ is an odd integer. (We are using here the fact that $\sigma M$ changes sign when the orientation is reversed, whereas $\chi M$ is independent of the orientation.)

We summarize the results on 2-fields in the following table. Here $M$ is a compact, orientable manifold, with a definite choice of orientation when $\operatorname{dim} M \equiv 0 \bmod 4$.

Table 1. The Index of a 2-Field

\begin{tabular}{c|c|c}
\hline \hline $\operatorname{dim} M$ \\
$(=m), m \geqq 5$ & $\pi_{m-1}\left(V_{m, 2}\right)$ & Index \\
\hline$m \equiv 1 \bmod 4$ & $Z_{2}$ & $k M$ \\
$m \equiv 2 \bmod 4$ & $Z \oplus Z_{2}$ & $(\chi M, 0)$ \\
$m \equiv 3 \bmod 4$ & $Z_{2}$ & 0 \\
$m \equiv 4 \bmod 4$ & $Z \oplus Z_{2}$ & $\left(\chi M,\left(\frac{1}{2}\left(\sigma M-(-1)^{k} \chi M\right)\right) \bmod 2\right)$ \\
& & \\
\hline
\end{tabular}

An important fact shown by Theorems 1-5 is that the index of a $k$-field, for $k=1$ and 2 and for the manifolds given in these theorems, is independent of the choice of $k$-field.

We emphasize at this point that Hopf's theorem holds even for 
nonorientable manifolds-using cohomology with local coefficients one shows that the index of a 1 -field is still given by the Euler characteristic (see $[65, \S 39.7]$ ). On the other hand, in Theorems $2-5$, we have assumed at least orientability of the manifold. Thus an outstanding problem is to compute the index of a 2 -field on a nonorientable manifold. But here an interesting fact emerges. The index of $a$ 2-field on an odd-dimensional nonorientable manifold is not necessarily independent of the choice of a particular 2-field. In fact one can prove

THEOREM 6. Let $M$ be a compact m-manifold, where $m$ is odd and $m \geq 5$, such that $\left(w_{1} M\right)^{2} \neq 0$. Suppose that $M$ admits a 2-field with finite singularities. Then for any class $u \in H^{m-2}\left(M ; Z_{2}\right)$ one can find a 2 -field $\left(X_{1}, X_{2}\right)$ with finite singularities such that

$$
\text { Index }\left(X_{1}, X_{2}\right)=\left(w_{1} M^{2} \cdot u\right)[M] .
$$

In particular, both 0 and 1 will occur as the index of a 2-field.

(If $M$ is an $m$-manifold and $v \in H^{m}\left(M ; Z_{2}\right)$, we let $v[M]$ denote the value of the class $v$ on the $\bmod 2$ generator of $H_{m}\left(M ; Z_{2}\right)$.)

On the other hand, if $\left(w_{1} M\right)^{2}=0$, one can show that the index of a 2-field is independent of the choice of field. Thus we have

Problem 1. Let $M$ be a compact odd-dimensional nonorientable manifold such that $\left(w_{1} M\right)^{2}=0$. Compute the mod 2 integer which is the index for any 2-field on $M$ with finite singularities.

The case of 2-fields on even-dimensional nonorientable manifolds seems much more difficult, because in the fibre bundle required for the problem the fundamental group of the base acts nontrivially on the fiber.

Problem 2. Let $M$ be a compact even-dimensional nonorientable manifold and suppose that $M$ has a 2-field with finite singularities.

(a) Determine whether the index is independent of the choice of 2-field.

(b) Compute the $Z_{2}$-index of a 2-field.

When $\operatorname{dim} M \equiv 1 \bmod 4$ we have had to require that $M$ be a spin manifold in order to compute the index of a 2-field. Thus for this dimension one has the following simpler version of Problem 1.

Problem 3. Let $M$ be a compact $m$-manifold, where $m \equiv 1 \bmod 4$. Suppose that $w_{1} M=0$ but that $w_{2} M \neq 0$. Compute the index of a 2-field on $M$ with finite singularities. (One can show that the index is independent of the choice of 2-field.)

Remark (AdDed April 2, 1969). This problem has now been solved by M. Atiyah. See the remark following Theorem 3.

Low DIMENSIONAL MANIFOLDS. So far we have assumed that our 
manifolds have dimension at least five. We now consider lower dimensions.

Dimension $M=2$. It is easily shown that $M$ has a 2 -field with finite singularities if and only if $w_{1} M=0$. Suppose this is the case. The index of such a 2 -field then lies in $\pi_{1}(\mathrm{SO}(2)) \approx Z$, and a simple argument shows that the index is given by the Euler characteristic of $M$.

Dimension $M=3$. One can show that $M$ has a 2 -field with finite singularities if and only if $\left(w_{1} M\right)^{2}=0$. The index of a 2-field takes values in $\pi_{2}\left(V_{3,1}\right)\left(\approx \pi_{2}(\mathrm{SO}(3))\right.$, which is zero.

In particular, every orientable 3-manifold is parallelizable (i.e., has a 3-field), a result due to Stiefel [68].

Dimension $M=4$. The index of a 2 -field here (assuming $M$ orientable) has been computed by Hirzebruch-Hopf [26]; their results will be discussed in $\$ 3$.

2. The span of a manifold. We now shift our emphasis away from the index of a $k$-field, and consider instead simply the question of whether a manifold admits a $k$-field without singularities. In other words, for $k>2$ we will be less concerned with computing the index of a $k$-field than with finding sufficient conditions for the index to vanish. To facilitate the discussion we make the following definition.

Definition 1. We define the span of a manifold $M$ to be the maximal number of linearly independent vector fields on $M$.

Thus span $M \geqq k$ if and only if $M$ has a $k$-field without singularities. The central problem of $k$-field theory is the following

Problem 4. Express the span of a manifold in terms of "familiar" algebraic invariants of the manifold.

The notion of a "familiar" invariant is, of course, relative; we give a list of some invariants at the end of the section. Also we propose several conjectures related to Problem 4.

Throughout this section we restrict attention to compact manifolds; very little is known in the noncompact case.

We wish to relate the material on the index, given in the preceding section, to the problem of determining the span of a manifold. To do this we need to know when a manifold has a $k$-field with finite singularities (see (1) in \$1). More generally, suppose that $M$ has been triangulated so as to be a simplicial complex. We then ask: When does there exist a $k$-field over the $i$-skeleton of $M, M^{i}, 1 \leqq i \leqq \operatorname{dim} M$ ? The relevant facts are these $[65, \S 38]$.

Let $m=\operatorname{dim} M$ and choose $k$ so that $1 \leqq k \leqq m$.

Fact 1. The Stiefel manifold $V_{m, k}$ is $(m-k-1)$-connected. Thus $M$ has a $k$-field over $M^{m-k}$. 
Fact 2. Suppose that $k \geqq 2$. Then $M$ has a $k$-field over $M^{m-k+1}$ if and only if

$$
\begin{array}{ll}
w_{m-k+1} M=0 & \text { if } m-k \text { odd, } \\
\delta^{*} w_{m-k} M=0 & \text { if } m-k \text { even and } M \text { orientable. }
\end{array}
$$

Here $\delta^{*}$ denotes the Bockstein cohomology coboundary operator associated with the exact sequence

$$
0 \rightarrow Z \rightarrow Z \rightarrow Z_{2} \rightarrow 0,
$$

and so $\delta^{*} w_{m-k} M \in M^{m-k+1}(M ; Z)$. (If $m-k$ even and $M$ is nonorientable, a similar result holds using a twisted Bockstein operator; see [65], [61]. Also, in the literature $\delta^{*} w_{2 i} M$ is often written as $\left.W_{2 i+1} M.\right)$

Now it is a striking fact, due basically to $\mathrm{Wu}[86]$, that certain Stiefel-Whitney classes of a manifold always vanish. Specifically, one can show (see $[40],[41]$ )

Fact 3 . Let $M$ be a compact, orientable $m$-manifold, $m \geqq 3$. Then,

$$
\begin{aligned}
w_{m-1} M=0 & \text { if } m=3 \bmod 4, \\
\delta^{*} w_{m-2} M=0 & \text { if } m \text { even. }
\end{aligned}
$$

If $M$ is compact and $\operatorname{dim} M=m$, then the $(m-1)$-skeleton of $M$ has the homotopy type of the space obtained by removing one point from the interior of each $m$-simplex of $M$. Thus, combining Facts 2 and 3, we have:

Let $M$ be a compact, orientable $m$-manifold, $m \geqq 3$. Then $M$ has a 2-field with finite singularities if $m$ is even, or if $m \equiv 3 \bmod 4$, or if $m$ $\equiv 1 \bmod 4$ and $w_{m-1} M=0$.

We now can use Table 1 in $\$ 1$ to determine whether span $M \geqq 2$ for $M$ a compact, orientable manifold.

TABLE 2. SPAN $M \geqq 2$

\begin{tabular}{l|c}
\hline \hline $\operatorname{dim} M(=m)$ & $\begin{array}{c}\text { Necessary and sufficient } \\
\text { conditions for Span } M \geqq 2\end{array}$ \\
\hline$m \equiv 1 \bmod 4$ & $w_{m-1}=0, k M=0$ \\
$m \equiv 2 \bmod 4$ & $x M=0$ \\
$m \geqslant 3 \bmod 4$ & $M$ always has span $\geqq 2$ \\
$m \equiv 0 \bmod 4, m>4$ & $\chi M=0, \sigma M \equiv 0 \bmod 4$ \\
\hline
\end{tabular}

One can continue for a ways in this fashion, giving necessary and sufficient conditions for $M$ to have span $\geqq 3$, etc., and we indicate such results later in the section. However, at this point we give examples of manifolds for which Problem 4 has been completely solved.

We consider first the $n$-dimensional sphere, $S^{n}$, for $n=1,2, \cdots$. 
Now for $n$ even $\chi S^{n} \neq 0$ and so by the Hopf theorem, Span $S^{n}=0$. We suppose then that $n$ is odd.

For each odd positive integer $n$, we define the Hurwitz-Radon number $h(n)$ as follows:

Write

$$
n+1=(2 a+1) \cdot 2^{c+4 d}
$$

where $a, c, d \geqq 0$ and $c \leqq 3$. Define

$$
h(n)=2^{c}+8 d-1 .
$$

Theorem 7 (Hurwitz-Radon). For $n$ odd, Span $S^{n} \geqq h(n)$.

The proof of the theorem occurs in two parts: the first by Hurwitz in [28], [29], and the second by Radon in [55]. For a modern proof (using group representations) see Eckmann [12].

A few scattered results on Span $S^{n}$ were obtained in the 1930's and 1940's (e.g., see [11], [82 ], [83]). The next result of generality was proved by Steenrod and J. H. C. Whitehead in 1951 [66].

Theorem 8 (Steenrod-J. H. C. Whitehead). Let $n$ be an odd integer, and write

$$
n+1=(\text { odd integer }) \cdot 2^{q} .
$$

Then, Span $S^{n}<2^{q}$.

We say that an $n$-manifold $M$ is parallelizable if Span $M=n$. Theorem 8 implies: If $S^{n}$ is parallelizable, then $n=2^{q}-1$. Classically, one knows that $S^{1}, S^{3}$ and $S^{7}$ are all parallelizable. The solution to the parallelizability of spheres was given in 1958 independently by Kervaire [32] and Milnor [44], using deep results of Bott [8].

Theorem 9 (Kervaire, Milnor). The sphere $S^{n}$ is parallelizable if and only if, $n=1,3$, or 7 .

Finally, the complete solution to the problem of Span $S^{n}$ was given by Frank Adams in 1962 [2] (see also [30]).

Theorem 10 (Adams). Let $n$ be an odd integer. Then

$$
\operatorname{Span} S^{n} \leqq h(n) .
$$

Therefore, by Theorem 8,

$$
\operatorname{Span} S^{n}=h(n) .
$$

To give the proof, Adams defined cohomology operations for a new cohomology theory, $K$-theory, developed by Atiyah and Hirzebruch [4]. 
Thus Problem 4 is solved for $S^{n}$; the only invariant required is the dimension, $n$.

The standard representation of $S^{n}$ as the space of unit vectors in $R^{n+1}$ gives an immersion of $S^{n}$ in $R^{n+1}$, with a trivial normal bundle. In general we call an $n$-manifold $M$ a $\pi$-manifold if there is an immersion of $M$ in $R^{n+1}$ with trivial normal bundle. It turns out that Problem 4 has been solved for $\pi$-manifolds.

ThEOREM 11. Let $M$ be a compact $\pi$-manifold of dimension $n \geqq 1$.

(a) Either $M$ is parallelizable or Span $M=\operatorname{Span} S^{n}$.

(b) If $n$ is even, then $M$ is parallelizable if and only if $\chi M=0$.

(c) If $n$ is odd and $n \neq 1,3$, or 7 , then $M$ is parallelizable if and only if $\widehat{\chi}_{2} M=0$.

Part (a) is proved in [10] and, by a somewhat different method, in [73]. Parts (b) and (c) are due to Kervaire [31], with a different proof given by Bredon-Kosinski in [10].

A theorem of Hirsch [23] enables one to formulate an equivalent definition of a $\pi$-manifold: $M$ is a $\pi$-manifold if and only if the tangent bundle of $M$ becomes trivial by taking the Whitney sum of it with a trivial line bundle. This implies that if $M$ is a $\pi$-manifold, we obtain a parallelizable manifold by removing a single point from $M$. And so we take this as the defining property for a broader class of manifolds than $\pi$-manifolds. We call a (compact) manifold $M$ almostparallelizable if we obtain a parallelizable manifold by removing a single point from $M$. To tell whether an almost-parallelizable manifold is in fact a $\pi$-manifold, we need a new set of invariants, the Pontrjagin classes. These are integral cohomology classes

$$
P_{k} M \in B^{4 k}(M ; Z), \quad k \geqq 0 .
$$

The relation between the Pontrjagin classes and span $M$ is the following.

Fact 4. Let $M$ be an $n$-manifold and let $i$ be a positive integer such that $4 i \leqq n$. If $P_{i} M \neq 0$, then Span $M \leqq n-2 i$.

See [30] and [43] for a discussion of these classes.

I am indebted to D. Frank for pointing out to me the following result.

Let $M$ be an almost-parallelizable $n$-manifold. Then $M$ is not a $\pi$-manifold if and only if $n \equiv 0 \bmod 4$ and $P_{k} M \neq 0$, where $n=4 k$.

Consequently, the following result, together with Theorem 11, essentially solves Problem 4 for almost-parallelizable manifolds.

Theorem 12. Let $M$ be an almost-parallelizable manifold of dimension $4 k, k>4$, such that $P_{k} M \neq 0$ and $\chi M=0$. Then Span $M=2 k-1$ or $2 k$. 
Proof. Let $D$ denote a small closed $4 k$-disk in $M$. If we collapse $M-D$ to a point, we obtain a $4 k$-sphere; let $p: M \rightarrow S^{4 k}$ denote the collapsing map. By hypothesis the tangent bundle of $M, \tau_{M}$, is trivial over $M-D$. Thus there is a $4 k$-plane bundle $\xi$ on $S^{4 k}$ such that

$$
\tau_{M}=p^{*} \xi .
$$

Since $P_{k} M \neq 0$, we have by Fact 4 that Span $M \leqq 2 k$. Now the Pontrjagin classes of a manifold are, by definition, the Pontrjagin classes of the tangent bundle $\tau M$. And by naturality of these classes, $P_{k} M$ $=p^{*} P_{k} \xi$, which means that $P_{k} \xi \neq 0$. For such bundles over $S^{4 k}$, Barratt and Mahowald (see [6] and [38]) have shown that the bundle has precisely $2 k-1$ linearly independent cross-sections. But relation $\left(^{*}\right)$ implies that Span $M \geqq$ no. of linearly independent crosssections in $\xi=2 k-1$. Thus, Span $M=2 k-1$ or $2 k$, as claimed.

Apart from these results on almost-parallelizable manifolds (including, of course, the particular cases of the spheres and $\pi$-manifolds), no solution of Problem 4 has been given for a large class of manifolds. The following two results enable one to give an upper bound to Span $M$ for certain manifolds.

Let $k$ be a positive integer. We say that a manifold $M$ is $k$ connected $\bmod 2$ if $H_{i}\left(M ; Z_{2}\right)=0$, for $0<i \leqq k$.

THEOREM 13. Let $n$ be an odd, positive integer, and write

$$
n+1=(\text { odd integer }) \cdot 2^{q} .
$$

Suppose that $M$ is a compact $n$-manifold which is $2^{q-1}$-connected $\bmod 2$ and with $w_{2^{q}} M=0$. If Span $M \geqq 2^{q}$, then $\hat{\chi}_{2} M=0$.

The proof is given in [14]. Since $\hat{\chi}_{2} S^{n} \neq 0$, the theorem is a generalization of Theorem 8 .

Consider now oriented manifolds $M$ with $\operatorname{dim} M \equiv 0 \bmod 4$; recall that $\sigma M$ denotes the signature of $M$ [24]. Given a positive integer $r$, define an integer $a_{r}$ as follows:

\begin{tabular}{l|l|l|l|l|l|l|l|l}
$r$ & 1 & 2 & 3 & 4 & 5 & 6 & 7 & 8 \\
\hline$a_{r}$ & 1 & 2 & 4 & 8 & 8 & 8 & 8 & 16
\end{tabular}

and $a_{r+8}=16 a_{r}, r \geq 1$.

Theorem 14 (MAyer-Frank). Let $M$ be a compact, oriented $4 k$ manifold, $k>1$, and let $r$ be a positive integer. If span $M \geq r$, then

$$
\sigma M \equiv 0 \bmod 2 a_{r} .
$$


This result can be obtained using the theorems of Mayer in $\$ 3$ of [41a]. (I am indebted to M. Atiyah for pointing this out.) D. Frank has obtained a similar result using a slightly different approach.

Conjectures on SPAN $M$. Let $n$ and $k$ be integers with $2 \leqq k<n / 2$. Suppose that $M$ is an orientable $n$-manifold with the following properties:

(A) $M$ is at least $(k-2)$-connected.

(B) $w_{k-1} M=0, w_{k} M=0$.

(C) If $n-k$ even, then $w_{n-k} M=0$. If $n-k$ odd, then $\delta^{*} w_{n-k-1} M=0$. For manifolds satisfying these conditions, the following conjecturesrelating Span $M$ to the integer $k$-seem reasonable.

$n$ odd.

ConjeCture 1. If $k \leqq \operatorname{Span} S^{n}$, then Span $M \geqq k$.

Conjecture 2. If $k>\operatorname{Span} S^{n}$, then $\operatorname{Span} M \geqq k$ if and only if $\hat{\chi}_{2} M$ $=0$.

$n$ even.

Conjecture 3. If $n \equiv 2 \bmod 4$, then Span $M \geqq k$ if and only if $\chi M=0$.

ConjeCtURe 4. If $n \equiv 0 \bmod 4, n>4$, then Span $M \geqq k$ if and only if $\chi M=0$ and $\sigma M \equiv 0 \bmod 2 a_{k}$, where $a_{k}$ is the integer given above Theorem 14.

As justification for the conjectures, they are known to be true in the following cases:

(i) $\pi$-manifolds, by Theorem 11 .

(ii) Almost-parallelizable manifolds of $\operatorname{dim} 4 k$ with $P_{k} \neq 0$, by Theorem 12.

(iii) Since $2^{q}>\operatorname{Span} S^{n}$, Theorem 13 is a weak corroboration of one implication in Conjecture 2.

(iv) Conjecture 4 is stated to fit precisely the results given in Theorems 5 and 14.

(v) Finally, for low values of $k$, the conjectures have been verified as follows [74], [75], [76], [81]:

Conjecture 1. $n \equiv 3 \bmod 8, k=2,3$;

$$
n \equiv 7 \bmod 8, k=2,3,4,5,6 \text {. }
$$

CONJECTURE 2. $n \equiv 1 \bmod 4, k=2,3$;

$$
n \equiv 3 \bmod 8, k=4,5,6 \text {. }
$$

Conjecture 3. $n \equiv 2 \bmod 4, k=2,3,5,6$.

CONJECTURE 4. $n \equiv 0 \bmod 4, k=2 ; n \equiv 0 \bmod 8, k=3,4$;

$n \equiv 0 \bmod 16, k=5$.

Of the four conjectures, the first is the most likely to be true. I will be quite surprised if it should be proved false. Conjecture 2 has been 
verified only for low values of $k$, where Theorem 13 still appliesthat is, if

$$
n+1=(\text { odd integer }) \cdot 2^{q},
$$

with $1 \leqq q \leqq 3$, then Span $S^{n}=2^{q}-1$. On the other hand, if $n=32 k$ $+15, k \geqq 0$, then $\operatorname{Span} S^{n}=8$. Thus, the first place to test Conjecture 2 is the following

Problem 5. Let $M$ be a compact 7-connected n-manifold, with $n \equiv 15$ $\bmod 32$. Suppose that $w_{8} M=0, w_{n-9} M=0$ and that $\hat{\chi}_{2} M=0$. Determine whether or not Span $M \geqq 9$.

Conjecture 2 would imply that Span $M \geqq 9$.

Conjecture 3 is made simply on the basis of results obtained so far; if it is false, some interesting new invariants of manifolds should play a role.

REMARKs. 1. Hypothesis (B) is frequently redundant, given hypothesis (A). For by $\mathrm{Wu}$ [85], unless $k-1$ (respectively $k$ ) is a power of two, one always has $w_{k-1} M=0$ (respectively, $w_{k} M=0$ ) since by (A), $w_{1} M=\cdots=w_{k-2} M=0$. Also, work of Strong [69] shows that if $k$ is greater than nine, then one always has $w_{k-1} M$ $=w_{k} M=0$, given $(\mathrm{A})$.

2. The conjectures given here are a revision of conjectures given in [81]. In particular, Conjecture 4 is new, based on the work of Frank.

Stronger conjectures. It is quite possible that some of the conjectures are true with weaker hypothesis than those given in (A)-(C). An example is to assume simply

$\left(\mathrm{A}^{\prime}\right) \quad M$ is $(k-2)$-connected mod 2.

In fact the results given under $(\mathrm{v})$, are proved assuming $\left(\mathrm{A}^{\prime}\right)$ at most.

An even stronger conjecture is to make no connectivity assumption on $M$, and replace (B) simply by

(B') $w_{1} M=\cdots=w_{k} M=0$.

I think it highly likely that Conjecture 1 remains true under these hypotheses, and so I make this a separate conjecture.

CONJECTURE 5. Let $M$ be a compact $n$-manifold, $n$ odd, and let $k$ be a positive integer such that $k \leqq \operatorname{Span} S^{n}$. If $w_{1} M=\cdots=w_{k} M=0$, then Span $M \geqq k$.

Notice that hypothesis (C) has been omitted. The reason is that one can prove quite easily, using the theorem of $\mathrm{Wu}$ [86], that:

Fact 5 . Suppose that $M$ is a compact $n$-manifold such that

$$
w_{1} M=\cdots=w_{2^{r}} M=0,
$$


where $r$ is an integer such that $1 \leqq 2^{r} \leqq n$. Let $k$ be the unique integer such that

$$
0 \leqq k<2^{r+2}, \quad n \equiv k \bmod 2^{r+2} .
$$

Then,

$$
w_{n-i} M=0 \quad \text { for } 0 \leqq i<k \text { and } \delta^{*} w_{n-k} M=0 .
$$

Notice that by Fact 5 , hypothesis (C) is not needed for Conjecture 1. Conjecture 5 has been verified for $k=2$ (see Table 2) and for $k=3$, as we now state.

THEOREM 15. Let $M$ be a compact spin manifold with $\operatorname{dim} M \equiv 3$ $\bmod$ 4. Then $\operatorname{span} M \geqq 3$.

The proof is given in [75].

On the other hand, it is not possible to rephrase Conjecture 2 using only hypothesis (B), as the following example shows.

Let $n=2^{q}-1$, where $q \geqq 5$, and consider real projective $n$-space, $P^{n}$. One has that

$$
w_{i} P^{n}=0, \quad \text { for } i \geqq 1
$$

and an easy calculation shows that $\hat{\chi}_{2} P^{n}=0$. On the other hand Span $P^{n}=$ Span $S^{n}$.

Low dimensional manifolds. So far in discussing Problem 4, we have assumed that our manifolds have arbitrary dimension. It turns out that if we consider only manifolds of dimension $\leqq 7$, a complete solution to Problem 4 can be given for most such manifolds. The only restrictions needed are:

(a) the manifolds are orientable,

(b) in some cases one needs spin manifolds,

(c) in other cases one needs to assume the $H^{4}(M ; Z)$ is without torsion.

For the details, see [79].

A List of invariants. In Problem 4 we asked for an expression of Span $M$ in terms of familiar algebraic (and numerical) invariants of $M$. For the results obtained so far, we have used the following invariants:

the dimension of $M$,

the Euler characteristic of $M, \chi M$,

the mod 2 semicharacteristic, $\hat{\chi}_{2} M$,

the real Kervaire semicharacteristic, $k M$,

the signature of $M, \sigma M$,

the Stiefel-Whitney classes of $M, w_{i} M$,

the Pontrjagin classes of $M, P_{i} M$.

Also, the Hurwitz-Radon numer $h(n)$ is referred to in Conjectures 
1-5. An interesting question is whether the solution of Problem 4 will ultimately require more invariants than those listed above.

3. Fields of tangent $k$-planes. Suppose that a manifold $M$ admits a $k$-field without singularities, say $\left(X_{1}, X_{2}, \cdots, X_{k}\right)$. At each point $x$ in $M$, the tangent vectors $X_{1}(x), \cdots, X_{k}(x)$ then span a $k$-dimensional linear subspace of the tangent space of $M$ at $x$. Thus the $k$-field gives rise to a field of tangent $k$-planes on $M$. Moreover an ordering of the vectors in the $k$-field gives rise to a natural orientation for the $k$-plane field. In this section we consider the question: When does a manifold admit a field of oriented tangent $k$-planes?

Notice that an oriented $k$-plane field defines an oriented $k$-plane bundle, which is a sub-bundle of the tangent bundle of $M$; and conversely. Moreover, if we assume $M$ has been given a Riemannian metric then a $k$-plane sub-bundle of $\tau_{M}$ has a naturally defined complementary bundle of dimension $m-k(m=\operatorname{dim} M)$, which we call the normal bundle to the $k$-plane field. (This normal bundle will play an important role in the following section.)

As with vector $k$-fields, one can define a $k$-plane field with finite singularities. The method of $\$ 1$ then associates with such a field an index which is an element of $\pi_{m-1}\left(G_{m, k}\right)$. (Here we are assuming that $m=\operatorname{dim} M$ and we let $G_{m, k}$ denote the Grassmann manifold of oriented $k$-dimensional linear subspaces of $R^{m}$. See [65].)

Now a field of oriented 1-planes is simply a tangent 1-field on $M$, which we have already considered. Thus we restrict attention to oriented $k$-plane fields with $k \geqq 2$. For simplicity we will leave off the word "oriented," though this will be assumed throughout the section.

We shift the problem slightly, as follows. Let $\eta$ be an (oriented) bundle over a manifold $M$, where $\operatorname{dim} \eta<\operatorname{dim} M$.

Question 1. Does $\eta$ give rise to a $k$-plane field on $M(k=\operatorname{dim} \eta)$ ? That is, is $\eta$ isomorphic to a sub-bundle of $\tau M$ ?

By considering a particular bundle $\eta$ it turns out that the fibration involved has fiber the Stiefel manifold $V_{m, k}$ rather than the Grassmann manifold $G_{m, k}(m=\operatorname{dim} M)$. But this is a great advantage, since $V_{m, k}$ is $(m-k-1)$-connected, while $G_{m, k}$ is just 1-connected [65] (see [78] for details). In particular, the bundle $\eta$ gives a $k$-plane field on the $(m-k)$-skeleton of $M$. We now compute the obstruction to $\eta$ giving a $k$-plane field on $M^{m-k+1}$; this will be a class in

$$
H^{m-k+1}\left(M ; \pi_{m-k}\left(V_{m, k}\right)\right) \text {. }
$$

Let $\xi$ be a vector bundle over a complex $X$. We denote by $[\xi]$ the stable bundle determined by $\xi-$ i.e., $[\xi]$ is an element in the reduced real $K$-theory group $K \widetilde{O}(X)$ (see [4]). Since the Stiefel-Whitney 
classes are stable invariants we can consider the classes $w_{i}[\xi], i \geqq 0$. Now let $\xi$ and $\eta$ be two bundles over $X$. We set

$$
\theta_{i}(\xi, \eta)=w_{i}([\xi]-[\eta]), \quad i>0,
$$

where the minus sign is taken in the group $K \widetilde{O}(X)$. If $X$ is a manifold $M$, we set

$$
\theta_{i}(\eta)=\theta_{i}\left(\tau_{M}, \eta\right), \quad i \geqq 0 .
$$

THEOREM 16. Let $M$ be a compact orientable m-manifold and let $\eta$ be an oriented $k$-plane bundle over $M, 1<k<m$. Then $\eta$ gives a $k$-plane field over the $(m-k+1)$-skeleton of $M$ if and only if

$$
\begin{gathered}
\theta_{m-k+1}(\eta)=0, \quad m-k \text { odd }, \\
\delta^{*} \theta_{m-k}(\eta)=0, \quad m-k \text { even. }
\end{gathered}
$$

The proof is given in [78, Theorem 1.4].

For the rest of the section we restrict attention to the case $m$ even and $k=2$. One then has the result

THEOREM 17. Let $M$ be an orientable $m$-manifold, $m$ even, and let $\eta$ be an oriented 2-plane bundle over $M$. Then $\delta^{*} \theta_{m-2}(\eta)=0$ and so $\eta$ gives a 2-plane field with only finite singularities on $M$.

If $m=4$ the result is given in [26]. Also, if $\eta$ is trivial, then $\theta_{m-2}(\eta)$ $=w_{m-2} M$, and the result is given in [41]. For a proof in general, see $[77, \S 4]$.

Thus with each oriented 2-plane bundle $\eta$ over an even-dimensional manifold $M$ we can associate an index:

$$
\operatorname{Index}(\eta) \in \pi_{m-1}\left(V_{m, 2}\right) \quad(m \text { even }) .
$$

This group is as follows.

$$
\begin{aligned}
\pi_{3}\left(V_{4,2}\right) & \approx Z \oplus Z, \\
\pi_{m-1}\left(V_{m, 2}\right) & \approx Z \oplus Z_{2}, \quad m>4 .
\end{aligned}
$$

(See [50].)

2-PLANES ON 4-MANIFOLDS: THE WORK OF HIRZEBRUCH AND HoPF. Hirzebruch and Hopf have solved completely the problem of computing the index to a field of 2-planes with finite singularities on an orientable 4-manifold. As noted above, the index is given by a pair of integers. We proceed to describe their result.

Let $M$ be a compact, 4-dimensional oriented manifold. Let $H$ denote the free abelian group $H^{2}(M ; Z) /$ Torsion subgroup. The orientation gives an isomorphism $H^{4}(M ; Z) \approx Z$ and Poincaré duality then gives a symmetric nonsingular bilinear form 
The signature of $M, \sigma M$, is by definition the signature of $S$. For any such form $S$, one readily proves:

there is a class $w \in H$ such that

$$
S(w, x) \equiv S(x, x) \bmod 2 \text { for all } x \in H .
$$

Given one class $w$ satisfying $\left(^{*}\right)$, then a second class $w^{\prime} \in H$ satisfies $\left(^{*}\right)$ if and only if $w^{\prime}=w+2 y$, for some $y \in H$. Thus the set of vectors $w$ satisfying $\left(^{*}\right)$ is a coset $W$ of $H / 2 H$. We denote by $\Omega$ the set of integers $S(w, w)$, for $w \in W$. (In case $H=0$, we set $\Omega=\{0\}$.)

THEOREM 18 (HIRzEBRUCH-Hopf). Let $M$ be a compact, 4-dimensional oriented manifold. Then $M$ has a field of 2-planes with finite singularities. The index of such a field is given by a pair of integers $(a, b)$. The following integers, and only these, occur as the index for some 2-plane field on $M$ :

$a=\frac{1}{4}(\alpha-3 \sigma-2 \chi), \quad b=\frac{1}{4}(\beta-3 \sigma+2 \chi), \quad$ for arbitrary $\alpha, \beta \in \Omega$.

Here $\sigma=\sigma(M)$ (= signature of $M), \chi=\chi(M)(=$ Euler characteristic of $M)$. The theorem is proved in [26].

REMARK. Since $a$ and $b$ are integers, so must be the expressions (rearranging terms slightly)

$$
\frac{1}{4}(\alpha-\sigma)-\frac{1}{2}(\sigma+\chi), \quad \frac{1}{4}(\beta-\sigma)-\frac{1}{2}(\sigma-\chi) .
$$

Now $\chi \equiv \sigma \bmod 2$, since each is simply the $\bmod 2$ value of the second Betti number of $M$. Thus the fact that $a$ and $b$ are integers is equivalent to saying that

$$
\gamma \equiv \sigma \bmod 4, \quad \text { for all } \gamma \in \Omega \text {. }
$$

In fact, one has a stronger result

$$
\gamma \equiv \sigma \bmod 8, \quad \text { for all } \gamma \in \Omega \text {. }
$$

See $[25$, p. 90$]$ for a proof.

Addendum on 2-Fields. Hirzebruch and Hopf also compute (in [26]) the index of a 2-field with finite singularities on an orientable 4-manifold $M$. Since $\pi_{3}\left(V_{4,2}\right)=Z \oplus Z$, the index is again given by a pair of integers, $(a, b)$.

TheOREM 19 (HIRzeBRUCH AND HOPF). $M$ admits a 2-field with finite singularities. The following pairs of integers, and only these, occur as the index for such a 2-field:

$$
a=\frac{1}{4}(\alpha-3 \sigma-2 \chi), \quad b=\frac{1}{4}(\alpha-3 \sigma+2 \chi),
$$

for all $\alpha \in \Omega$. 
2-PLANE FIELDS ON COMPACT ORIENTABLE $m$-MANIFOLDS, $m$ EVEN AND $>4$. Let $\eta$ be a 2-plane field with finite singularities on $M$, where $\operatorname{dim} M=m, m$ even and $>4$. The index of $\eta$ is then an element of a group isomorphic to $Z \oplus Z_{2}$, and so has two components: the $Z$-index and the $Z_{2}$-index. We are able to give a complete computation of the $Z$-index; the $Z_{2}$-index is known only in certain cases.

Now an oriented 2-plane bundle $\eta$ over a complex $X$ is completely determined by its Euler class $\chi(\eta) \in H^{2}(X ; Z)$. This is because the classifying space $B S O(2)$ is an Eilenberg-MacLane $K(Z, 2)$-space. Suppose that $X$ is an orientable manifold $M$ of $\operatorname{dim} 2 q$ and let $u$ be a class in $H^{2}(M ; Z)$. Define

$$
\theta(u)=\sum w_{2 j} M \cup u^{i} \in H^{2 q-2}\left(M ; Z_{2}\right)
$$

where the summation is taken over all nonnegative integers $i$ and $j$ such that $i+j=q-1(\operatorname{dim} M=2 q)$. One easily checks that

$$
\theta(u)=\theta_{2 q-2}(\eta),
$$

where $\eta$ is the oriented 2-plane bundle with $\chi(\eta)=u$. Thus, by Theorem $17, \delta^{*} \theta(u)=0$-i.e., $\theta(u)$ is the mod 2 reduction of a class in $H^{2 q-2}(M ; Z)$.

Given classes $v \in H^{r}(M ; Z)$ and $w \in H^{*}(M ; Z)$, where $r+s=\operatorname{dim} M$, define an integer $\Gamma(v, w)$ by

$$
\Gamma(v, w)=(v \cup w) \cdot[M],
$$

where we now suppose that $M$ is oriented, and $[M]$ denotes the integral generator in $H_{2 q}(M ; Z)$, corresponding to the orientation.

Our result is (see [77])

THEOREM 20. Let $M$ be an m-dimensional oriented manifold, where $m$ is even and $>4$, and let $u$ be a class in $H^{2}(M ; Z)$. Then the following integers, and only these, occur as the $Z$-index of 2-plane fields on $M$ with finite singularities, and with Euler class $u$,

$$
\chi_{M}-\Gamma(u, v),
$$

where $v$ runs over all classes in $H^{m-2}(M ; Z)$ such that $v \bmod 2=\theta(u)$.

Notice that $\Gamma(u, v)=-\Gamma(-u, v)$, and so the set of integers occurring in the theorem is independent of the choice of orientation for $M$.

In general we are unable to compute the $Z_{2}$-index of a 2-distribution $\eta$ with finite singularities. However, one can show [77, Theorem 1.3] that if $\operatorname{dim} M \equiv 2 \bmod 4$ and if $\chi(\eta) \equiv 0 \bmod 2$, then the $Z_{2}$-index of $\eta$ is zero and so we have 
THEOREM 21. Let $M$ be an oriented $m$-manifold, where $m \equiv 2 \bmod 4$, and let $u \in H^{2}(M ; Z)$. Then there exists a 2-plane field (without singularities) on $M$, with Euler class $2 u$, if and only if there is a class $v \in H^{m-2}(M ; Z)$ such that

$$
u \bmod 2=w_{m-2}(M) \text {, and } 2 \Gamma(u, v)=\chi M .
$$

We have used here the fact that $\theta(2 u)=w_{m-2} M$.

To give an example, it turns out that Theorems 20 and 21 suffice to classify 2-plane fields on complex projective space $C P^{m}$ (of $2 m$ real dimensions). Let $x \in H^{2}\left(C P^{m} ; Z\right)$ denote the canonical generator.

THEOREM 22. Let $m$ and $s$ be integers with $m \geqq 3$. Then there is a 2-plane field on $C P^{m}$ with Euler class $s x$ if and only if the following conditions are satisfied.

(i) $m$ is odd, say $m=2 q-1$;

(ii) $s$ is even;

(iii) there is an integer $t$ such that

$$
t \equiv q \bmod 2 \text { and } s t=2 q .
$$

Proof. We use the following easily derived facts.

(i) $\theta(x)=m x^{m-1} \bmod 2$.

(ii) $\theta(0)=\left(\begin{array}{l}m+1 \\ 2\end{array} x^{m-1} \bmod 2\right.$.

(Recall that $w_{2 i}\left(C P^{m}\right)=\left({ }_{2 i}^{m+1}\right) x^{i} \bmod 2$.) Suppose first that $m$ is even. By the above formulae, $\Gamma(u, v)$ is even for all $v$ such that $v \bmod 2$ $=\theta(u)$. But $\chi\left(C P^{m}\right)=m+1$ and so is odd in this case, so that $\chi M$ $-\Gamma(u, v) \neq 0$. Thus there can be no 2 -plane field when $m$ is even. Suppose then that $m$ is odd. If $s$ is odd, then by (i) $\theta(s x)=\theta(x) \not \equiv 0$ and so $\Gamma(s x, v)$ is an odd integer for all $v$ such that $v \bmod 2=\theta(s x)$. But $\chi\left(C P^{m}\right)$ is now even and so again $\chi M-\Gamma(u, v) \neq 0$. The result for $m$ odd and $s$ even follows from Theorem 21 .

Our lack of information about the $Z_{2}$-index leaves open the following problem.

Problem 6. Let $M$ be a compact orientable m-manifold, where $m$ is even and greater than five. Let $\eta$ be a 2-plane field on $M$ with finite singularities. Compute the $Z_{2}$-index of $\eta$. (If $M \equiv 2 \bmod 4$, assume that $\chi \eta \neq 0$ $\bmod 2$.)

ODD-DIMENSIONAL MANIFOLDS. For compact manifolds of dimension $4 k+3, k \geqq 0$, we have obtained a complete solution to the problem of 2-plane fields.

THEOREM 23. Let $M$ be an orientable $m$-manifold with $m \equiv 3 \bmod 4$ and let $\eta$ be an oriented 2-plane bundle over $M$. Then $\eta$ gives a 2-plane field on $M$ if and only if $\theta_{m-2}(\eta)=0$. In particular, if $w_{2} \eta=0$, then $\eta$ 
gives a field of 2-planes on $M$.

The proof is given in $[78, \S 1]$; a somewhat weaker result is also proved there for manifolds of dimension $4 k+1$.

OPEN QUESTIONS. We simply indicate a number of topics about which practically nothing is known:

(i) $k$-plane fields on nonorientable manifolds,

(ii) nonorientable $k$-plane fields on manifolds,

(iii) $k$-plane fields on orientable manifolds, with $k \geqq 3$.

Of course, there is the information on the first obstruction given in Theorem 16. Also, on topic (iii) a partial result for $k=3$ is given in $[78, \S 1]$. But otherwise, to my knowledge, nothing is known about these topics. In the light of Theorem 14 the following problem looks interesting.

Problem 7. Let $M$ be a compact oriented manifold with $\operatorname{dim} M \equiv 0$ mod 4. Suppose that $M$ admits a family of tangent $k$-planes, $k \geqq 2$. What then can be said about the signature of $M, \sigma M$ ?

REMARK (ADDED APRIL 2, 1969). M. Atiyah has recently informed me of research germane to Problem 7. For example, Atiyah shows that if $M$ admits a family of 2-planes, then $\chi M$ is even and $\chi M \equiv \sigma M$ mod 4. Also, if $M$ admits $r$ independent oriented plane fields, each with $\operatorname{dim} \equiv 1 \bmod 4$, then $\sigma M$ has divisibility properties similar to those given in Theorem 14 (for an $r$-field on $M$ ).

4. Foliations. Suppose that a manifold $M$ has a field of tangent $k$-planes. We now regard such a field as a section $s$ in the bundle with fiber $G_{m, k}$ associated to the tangent bundle of $M(m=\operatorname{dim} M)$. In particular, we say that the field is smooth if the section $s$ is a smooth map. Let $N$ be an injectively immersed submanifold of $M$. We say that $N$ is an integral submanifold of $s$ if for each point $x$ in $N$, the tangent space to $N$ at $x$ is the $k$-plane $s(x)$.

Definition 2. A smooth $k$-plane field $s$ is said to be completely integrable if through each point in $M$ there is an integral submanifold of $s$.

An integrable $k$-plane field is also called a foliation (this is equivalent to the local product condition of the classical definition [56]) and the maximal connected integral submanifolds are called leaves. The leaves of a foliation partition the manifold.

A fundamental result on foliations is the theorem of Frobenius [67, p. 132].

THEOREM OF Frobenius. Let $s$ be a smooth $k$-plane field on a manifold $M$. Then $s$ is completely integrable if and only if for every pair of 
vector fields $X$ and $Y$ on $M$, such that $X(x), Y(x) \in s(x)$ for all $x \in M$, one has $[X, Y](x) \in s(x)$.

Here $[X, Y]$ denotes the Lie bracket of vector fields [67]. There is a dual formulation of the theorem in terms of differential forms, [67, p. 134]. Using the theorem one can show, for example, that if $\pi: M$ $\rightarrow N$ is a smooth fiber bundle, then the fibres of $\pi$ are the leaves of a foliation of $M$. (The foliation is simply "the bundle along the fibres." See $[6, \S 7.4]$.)

We consider in this section some basic questions about foliations. Question 2. Suppose that a manifold $M$ has a $k$-plane field.

(a) (Reeb [56].) Does $M$ then admit a k-plane field that is completely integrable-i.e., a foliation?

(b) (Haefliger [17].) Under what conditions is the given k-plane field homotopic to a foliation?

(c) If $M$ has a $k$-dimensional foliation, then is every $k$-plane field on $M$ homotopic to a foliation?

Reeb remarks, in [56], that the answer to (a) is probably negative, in general. Using a recent result of Bott we will give an example where the answer is indeed negative. When $k=1$ (and $M$ is compact), (c) has an affirmative answer, by the classical existence theorem for ordinary differential equations (e.g., see [34]). We will discuss recent results of $\mathrm{J}$. Wood, which give an affirmative answer to (c) for $k=2$ and $M$ a compact 3-manifold-assuming that the normal bundle of the foliation is trivial. On the other hand, the recent work of Bott enables one to give examples where (c) has a negative answer. As to (b), it begins to appear that the normal bundle of the $k$-plane field may be the determining factor as to whether the field is homotopic to a foliation.

I am indebted to J. Wood for several useful conversations about the material in this section.

An $(n-k)$-plane field on an $n$-manifold will be called a plane field of codimension $k$. Most of the positive results on foliations concern plane fields of codimension one. Following Wood [84] we say that a manifold $M$ satisfies condition $\mathrm{H}$ if it has the following property.

Condition H. Every plane field of codimension one, with trivial normal bundle, is homotopic to a foliation.

Theorem 24 (Wood). Every compact 3-manifold satisfies Condition H.

(The existence of a foliation of codimension one on an orientable 3-manifold was proved by Lickorish [35] and Novikoff-Zieschang [47].) 
Wood also gives higher dimensional examples of manifolds satisfying condition $\mathrm{H}$.

THEOREM 25. Let $M$ be a compact manifold with a foliation $s$, of codimension 1, which has a trivial normal bundle, and let $C$ be a nonempty disjoint union of circles, each transverse to $s$, such that $H_{1}(C)$ $\rightarrow H_{1}(M)$ is onto. Then $M$ satisfies Condition $\mathrm{H}$.

The proof is given in [84, Theorem 9.4]. For example, consider the product $S^{1} \times M$. This has the product foliation, with leaves $x \times M$ for each $x \in S^{1}$. Wood shows that the condition given in Theorem 25 is satisfied, thus proving

Corollary 1. For any compact manifold $M$, the manifold $S^{1} \times M$ satisfies Condition $\mathrm{H}$.

For a second example, suppose that $M$ is a simply connected compact manifold, with vanishing Euler characteristic. Then $M$ has precisely two homotopy classes of vector 1-fields (assuming $\operatorname{dim} M$ $>3$ ), and hence two homotopy classes of plane fields of codimension one. Suppose that one of these classes contains a foliation. Using Theorem 25, Wood shows that the other class does also [84, Theorem 9.5], and so we have

Corollary 2. Any simply connected compact manifold with a foliation of codimension one satisfies Condition $\mathrm{H}$.

So far we have considered foliations on compact manifolds. For noncompact (i.e., open) manifolds Phillips' work on submersions [52] gives strong results on foliations. (A submersion from $M$ to $N$ is a smooth map $f$ which, at each point $x$ of $M$, maps the tangent space of $M$ at $x$ onto the tangent space of $N$ at $f(x)$.) Phillips shows that if an open manifold has a vector $k$-field, then this field is homotopic to the gradient $k$-field given by a submersion $M \rightarrow R^{k}$. (We assume here that $M$ has been given a Riemannian metric.) In particular, the plane field of codimension $k$ orthogonal to the vector $k$-field is homotopic to a foliation.

Theorem 26 (Phillips). Let $M$ be an open manifold. Then every $k$-plane field on $M$, with trivial normal bundle, is homotopic to a foliation.

Now every open manifold has a vector 1 -field and hence a plane field of codimension one with a trivial normal bundle. Thus, by Theorem 26, an open manifold satisfies Condition H. However, Phillips proves in addition (see [53]) that a plane field of codimension 
one is homotopic to a foliation, even if the normal bundle of the field is not trivial.

TheOREM 27 (PhilliPs). On an open manifold, if the structure group of the normal bundle of a plane field reduces to a discrete group, then the plane field is homotopic to a foliation. In particular, every plane field of codimension one is homotopic to a foliation.

CONJECTURES ON FOLIATIONS. On the basis of the results described above, I think that the following conjecture is reasonable.

CONJECTURE 6. Let $M$ be a compact manifold with a foliation of codimension one, such that the normal bundle of the foliation is trivial. Then every plane field of codimension one, with a trivial normal bundle, is homotopic to a foliation.

As to the existence of a foliation of codimension one, we consider the problem from the following point of view. Let $M$ be a compact manifold with $\chi M=0$ and let $M_{0}$ denote the open manifold obtained by removing a point from $M$. By Theorem $27, M_{0}$ has a foliation of codimension one, call it $s$. One can now try to follow the procedure given in $\$ 1$-attach to $s$ an algebraic "index" which measures whether or not $s$, restricted to some suitable subspace of $M_{0}$, can be extended, as a foliation, to all of $M$.

In spite of the uncertainty of getting positive results by this procedure, the following conjecture still seems to me to be reasonable, although not as likely as Conjecture 6 .

CONJECTURE 7. Every compact manifold with vanishing Euler characteristic has a foliation of codimension one.

The first place to test the conjecture is the odd-dimensional spheres.

Problem 8. Let $n$ be an odd integer greater than three. Does $S^{n}$ admit a foliation of codimension one?

A. Phillips has pointed out that no example is known, other than $S^{3}$, of a compact, simply connected manifold with a foliation of codimension one. Thus, as a generalization of Problem 8, we have

Problem 9. Give examples of compact, simply connected manifolds (of $\operatorname{dim}>3$ ), with a foliation of codimension one.

REMARK (ADDED IN PROOF). J. Wood has pointed out that examples can be constructed by taking the product of $S^{3}$ with a simply connected manifold. Thus, the problem really is to find other types of examples.

Nonexistence of foliations. The theorem of Bott. Up to now there has been no way to study foliations using the familiar algebraic invariants of topology. However, in a recent lecture, $R$. Bott an- 
nounced a theorem which gives an algebraic tool for showing the nonexistence of certain foliations.

Let $\xi$ be a vector bundle over a complex $X$. Denote by $R^{*}(\xi)$ $C H^{*}(X ; Q)$ the subring of the rational cohomology of $X$ generated by the Pontrjagin classes of $\xi$. (We regard each class $P_{i} \xi$ as a rational class via the natural inclusion $Z \subset Q$.) Notice that $R^{*}(\xi)=R^{*}\left(\xi \oplus \epsilon^{n}\right)$, where $\epsilon^{n}$ denotes the trivial bundle of dimension $n$. Thus $R^{*}(\xi)$ depends only upon the stable class of $\xi$.

THEOREM 28 (BotT). Let $M$ be a compact orientable manifold and let $\eta$ be an orientable $(n-k)$-plane field on $M$. Consider the quotient bundle $\tau_{M} / \eta$. If $\eta$ is homotopic to a foliation of $M$, then $R^{j}\left(\tau_{M} / \eta\right)=0$ for $j>2 k$.

Of course the theorem gives information only when $k \leqq \frac{1}{2} \operatorname{dim} M$ and when there is at least one class $P_{i}\left(\tau_{M} / \eta\right) \neq 0$. In particular, the theorem gives no information when $k=1$ - the case of codimension one that we have considered above.

In $\$ 3$ we showed the existence of certain 2-plane fields on $C P^{m}$, for $m$ odd. By putting a Riemannian metric on $C P^{m}$, one then obtains, for each 2-plane field, a complementary $(2 m-2)$-plane field. Using Bott's result, we will show that no such $(2 m-2)$-plane field can be a foliation (for $m>3$ ).

EXAMPLE 1. (a) For $m$ odd, $C P^{m}$ has plane fields of codimension 2, but no foliation of codimension 2 (assuming $m>3$ ).

(b) Let $M=S^{1} \times S^{1} \times C P^{m}$. Then $M$ has a foliation of codimension two. On the other hand, for $m \geqq 3, M$ has a plane field of codimension 2 which is not homotopic to a foliation.

Thus (a) and (b) provide negative answers to parts (a) and (c) of Question 2.

To see (a), let $\eta$ be a plane field of codimension 2 on $C P^{m}(=M)$ and let $\xi$ be a complementary 2-plane field:

$$
\tau_{M}=\xi \oplus \eta .
$$

Now $\chi_{M}=m+1 \neq 0$, and so $\chi \xi \neq 0$, since $\chi \tau_{M}=\chi \xi \chi \eta$. Thus $\chi \xi=s x$, $s \neq 0$, where $x$ generates $H^{2}\left(C P^{m}, Z\right)$. Since $\xi$ is an orientable 2-plane bundle, $P_{1} \xi=\chi(\xi)^{2}=s^{2} x^{2}$, and so

$$
P_{1}\left(\tau_{M} / \eta\right)=s^{2} x^{2} .
$$

Therefore, if $m>3$,

$$
\left(P_{1}\left(\tau_{M} / \eta\right)\right)^{2}=s^{4} x^{4} \neq 0,
$$


and so by Theorem $28, \eta$ can not be homotopic to a foliation. For (b), let $M=S^{1} \times S^{1} \times C P^{m}, m \geqq 3$. Then $\tau_{M}=\tau C P^{m} \oplus 2 \epsilon$, where $\epsilon$ denotes the trivial line bundle. Let $\zeta$ denote the orientable 2-plane bundle over $C P^{m}$ such that $\chi \zeta=x$. Then, (see $\left.[43]\right), \tau_{M}=(m+1) \zeta$. Let $\eta=m \zeta$. Then $\eta$ is a plane field of codimension two on $M$ with $P_{1}\left(\tau_{M} / \eta\right)$ $=x^{2}$. Thus by the same argument as above, $\eta$ can not be homotopic to a foliation. On the other hand, $M$ does have a foliation of codimension 2 given by the product decomposition $\left(S^{1} \times S^{1}\right) \times C P^{m}$.

Foliations of Dimension two. The positive results we have discussed so far have all been either in codimension one or dimension one. For foliations of $\operatorname{dim} k$, with $1<k<\operatorname{dim} M-1$, essentially nothing is known concerning Question 2, except now the information given by Theorem 28 . We now consider the case $k=2$, where Theorem 28 gives no information.

In $\$ 3$ we gave sufficient conditions for some manifolds to admit a 2-plane field-in particular we gave a complete classification of 2-plane fields (thought of simply as 2-plane bundles) on $C P^{m}$. Let $x \in H^{2}\left(C P^{m} ; Z\right)$ denote the canonical generator. Recall that for $m$ odd, there is always a 2-plane field with Euler class $2 x$.

EXAMPLE 2. For $m$ odd, $C P^{m}$ admits a 2-dimensional foliation with Euler class $2 x$.

Proof. Let $m=2 k+1$. Recall that one has a smooth fibration

$$
S^{2} \stackrel{i}{\rightarrow} C P^{2 k+1} \stackrel{\pi}{\rightarrow} Q P^{k}
$$

$[62$, p. 150]. Thus,

$$
\tau\left(C P^{2 k+1}\right)=\sigma^{2} \oplus \pi^{*} \tau\left(Q P^{k}\right),
$$

where $\sigma^{2}$ denotes "the bundle along the fibers" (see $[6,87.4]$ ). Since $i^{*} \sigma^{2}=\tau\left(S^{2}\right)$ and $\chi\left(S^{2}\right)=2$, the result follows from the fact that the bundle along the fibres, of a smooth fibration, is a foliation.

Now Theorem 22 shows that for $k>1, C P^{2 k+1}$ has a 2-plane field with Euler class $s x$, where $s>1$.

Problem 10. Let s be an integer greater than one such that $C P^{2 k+1}$ has a 2-plane field with Euler class sx. Determine whether $C P^{2 k+1}$ has a foliation with this Euler class.

Suppose that a compact manifold $M$ admits a vector 2-field $\left(X_{1}, X_{2}\right)$ (cf. $\left.\S \S 1,2\right) ;$ let $s$ denote the 2-plane field spanned by $\left(X_{1}, X_{2}\right)$. This seems to be an especially interesting type of 2-plane family to study, from the point of view of foliations. A possible conjecture is that every such (trivial) 2-plane field is homotopic to a foliation, but at present we really have no information on this question. One 
also can make the complementary conjecture: every plane field of codimension two, with trivial normal bundle, is homotopic to a foliation. By Theorem 26, the foliation has but a single point of singularity. Also, Theorem 28 imposes no conditions since the normal bundle is trivial.

4-Dimensional MANifolds. Let $M$ denote a compact, oriented 4-manifold, and let $M_{0}=M-p t . M_{0}$ has a 2-plane field $s$, which on $M$ is a 2-plane field with one singularity. Suppose that the 2-plane field on $M_{0}$ is integrable. We define the index of this foliation to be the index of the 2-plane field $s$, which is a pair of integers as given in Theorem 18. S. S. Chern has suggested the following problem.

Problem 11 (Chern). For which pairs of integers $(a, b)$, as given in Theorem 18, does $M_{0}$ have a 2-dimensional foliation with index $(a, b)$ ?.

Consider, for example, the complex projective plane $C P^{2}$. Set $P_{0}=C P^{2}-p t$. Using Theorems 18 and 26 one obtains:

The following pairs of integers occur as the index of a 2-dimensional foliation on $P_{0}$ :

$$
\left(k^{2}+k-2, k^{2}+k+1\right), \quad k \in Z .
$$

Foliations OF SPHERES. Reeb [56, p. 112] defined a foliation of codimension one on $S^{3}$, which has played a crucial role in subsequent work on foliations, (e.g., see [35], [84]). Now $S^{3}$ is parallelizable, which suggests that the next simplest manifold to study is $S^{7}$, which is also parallelizable. In particular $S^{7}$ has $k$-plane fields for $1 \leqq k \leqq 6$. As always, the line fields are integrable. From the fibration

$$
S^{3} \rightarrow S^{7} \rightarrow S^{4}
$$

one obtains a 3-dimensional foliation of $S^{7}$. Now $S^{3}$ has a foliation of dimension 2, but it is not clear whether this can be extended to give a foliation of $S^{7}$.

Problem 12. Determine whether $\mathrm{S}^{7}$ has foliations of dimensions 2, 4, 5, or 6. In particular is there a 2-dimensional foliation which gives a foliation of each fiber?

More generally, one has the fibration [65]

$$
S^{3} \rightarrow S^{4 k+3} \rightarrow Q P^{k},
$$

thus giving a foliation of dimension 3 of $S^{4 k+3}$.

Problem 13. There are precisely 4 homotopy classes of 2-plane fields on $S^{4 k+3}, k \geqq 1$. Determine which of these contain foliations.

This, of course, is a special case of Question 2 for $k=2, M=S^{4 k+3}$.

CoMmUting veCtoR FIELDS. Following Milnor [36] we say that a manifold $M$ has rank $\geqq k$ if there is a $k$-field $X_{1}, \cdots, X_{k}$ on $M$ such 
that

$$
\left\lfloor X_{i}, X_{j}\right]=0, \quad \text { for all } 1 \leqq i, j \leqq k \text {. }
$$

By the Frobenius Theorem if rank $M \geqq k$, then $M$ has a foliation of dimension $k$ spanned by the $k$-field. Also, the additive group $R^{k}$ acts differentiably on $M$ so that each orbit has dimension $k$.

Some results are known on rank $M$. For example [36],

THEOREM 29 (Lima). Rank $S^{3}=1$.

More generally, Novikoff has proved [47]

TheOREM 30 (NovikofF). If $M^{3}$ is a closed orientable manifold with noncontractible universal covering space, then rank $M=1$. Furthermore, if $\pi_{1} M^{n}$ is finite, or if $\pi_{2} M^{n} \neq 0$, then rank $M \leqq n-2$ for $n \geqq 3$.

See also [58], [59], [60].

For a manifold $M$ define the Euler-Poincaré polynomial by

$$
\chi_{M}(t)=\sum b_{i} t^{i}
$$

where $b_{i}$ is the $i$ th Betti number of $M$. Thus $\chi M=\chi_{M}(-1)$, and so $\chi M=0$ if and only if -1 is a root of $\chi_{M}(t)$.

Conjecture 8 (Lima). If rank $M>1$, then -1 is a multiple root of $\chi_{M}(t)$.

REMARK. I have recently been informed of the following result by H. Rosenberg, R. Roussarie, and D. Weil.

$A$ compact, orientable 3-manifold $M$ has rank 2 if and only if $M$ is a nontrivial torus bundle over the circle. Since all compact, orientable 3-manifolds have rank $\geqq 1$ and since the 3-torus (i.e., the trivial 2-torus bundle over the circle) has rank 3, the problem of determining the rank is solved for closed, orientable 3-manifolds.

Note that these results corroborate Conjecture 8 in dimension 3.

We now consider a somewhat more general notion than that of commuting vector fields. Let $\left(X_{1}, \cdots, X_{k}\right)$ be a $k$-field on a manifold $M$. Suppose there are constants $\gamma_{a b c}, 1 \leqq a, b, c, \leqq k$, such that

$$
\left[X_{a}, X_{b}\right]=\sum_{c} \gamma_{a b c} X_{c} \text {. }
$$

The symbols $X_{i}$, considered abstractly, generate a $k$-dimensional Lie algebra \&. A theorem of Palais [51, Corollary 2, p. 82] asserts that: there is a simply connected Lie group $G$ whose Lie algebra is isomorphic to $\mathcal{G}$, and a smooth action of $G$ on $M$ such that the orbits of this action have dimension $k$.

To put it differently, condition (*) implies, by the Frobenius 
Theorem, that the $k$-field spans a foliation. By Palais' Theorem, the leaves of this foliation are homeomorphic to $G \bmod$ a discrete subgroup.

Out of all this one obtains the following, rather grandiose, problem. Problem 14. Let $\left(X_{1}, \cdots, X_{k}\right)$ be a $k$-field on a manifold M. Determine whether or not the $k$-field gives a Lie algebra, as in (*). In particular, for $k=2$ and 3, find necessary conditions on $M$ (keeping in mind Theorem 30) for the k-field to give a nontrivial Lie algebra.

5. Methods. The theorems given in $\S \S 1-3$ fall under the general heading: determine whether a given fiber bundle has a cross-section. In these theorems the bundle is always one associated to the tangent bundle of a manifold-with fiber either the Stiefel manifold $V_{m, k}$ or Grassmann manifold $G_{m, k}$. Steenrod's book [65] gives the classical approach to obstruction theory for cross-sections to fiber bundles.

In recent years a different approach to obstruction theory has evolved, initiated by the work of Postnikov [54]. There are two important aspects to this more recent approach:

(i) the classifying bundle, and

(ii) the Postnikov resolution of a map.

We proceed to comment briefly on each of these.

We emphasize that, in what follows, only the homotopy-theoretic methods of $\S \S 1-3$ will be discussed. The results given in $\$ 4$ require rather different techniques, which will not be considered here.

The CLASSIFYing BUNdLe. Let $n$ be a fixed, positive integer and consider $n$-plane bundles over $\mathrm{CW}$-complexes. For such bundles one has a classifying bundle $\gamma_{n}$ (over a base space $B_{n}$ ) with the following property.

Fact 6. Given an $n$-plane bundle $\xi$ over a complex $X$ there is a map $f$ (unique up to homotopy) from $X$ to $B_{n}$ such that $\xi$ is equivalent to the bundle induced by $f$ from $\gamma_{n}, f^{*} \gamma_{n}$ (see [30]).

The space $B_{n}$ is the Grassmann space of $n$-dimensional linear subspaces in countably infinite dimensional Euclidean space. See [30], [43] for details.

Let $k$ be an integer with $0<k \leqq n$. With each $n$-plane bundle $\xi$, with projection $\pi: E \rightarrow X$, we associate a bundle $\xi(k)$, with projection $\pi(k): E(k) \rightarrow X$, and with fiber $V_{n, k}$. The space $E(k)$ is defined to be the space of all points $\left(x, v_{1}, \cdots, v_{k}\right)$, where $x \in X$ and where $v_{1}, \cdots, v_{k}$ are linearly independent vectors in the $n$-dimensional vector space $\pi^{-1}(x)$. The map $\pi(k)$ is simply the projection on the $x$-coordinate. Notice that $\xi$ has $k$ linearly independent cross-sections if and only if $\xi(k)$ has a single section. (In a similar fashion one can define the associated bundle with fiber $G_{n, k}$.) 
As above, suppose that $\xi$ is equivalent to the bundle $f^{*} \gamma_{n}$. Then $\xi(k)$ is equivalent to the bundle $f^{*} \gamma_{n}(k)$. Let $\pi_{n}(k): E_{n}(k) \rightarrow B_{n}$ denote the projection for $\boldsymbol{\gamma}_{n}(k)$. Then $f^{*} \gamma_{n}(k)$ (and hence $\xi(k)$ ) has a section if and only if there is a map $g: X \rightarrow E_{n}(k)$ such that $\pi_{n}(k) \circ g=f: X \rightarrow B_{n}$.

See $[30],[43]$ for details.

Summing up then, we have reduced the cross-section problem to the problem of lifting a map to the total space of the classifying bundle. We now turn to a study of this problem.

The Postnikov resolution of a map. We change the notation slightly. Let $\pi$ be a map from a space $T$ to a space $B$. Given a map $f$ : $X \rightarrow B$ we are now studying the problem: determine whether $f$ lifts to $T$-i.e., does there exist a map $g: X \rightarrow T$ such that $\pi g=f$ ? We begin by observing a situation where this problem has a simple solution.

For the rest of the section we suppose that all spaces have a basepoint (written $*$ ) and that maps and homotopies preserve basepoints. Let $C$ be a space; define the path space $P C$ to be the space of all maps $\pi: I \rightarrow C$ such that $\lambda(0)=* \in C$. Let $p: P C \rightarrow C$ be the map $\lambda \rightarrow \lambda(1)$. Then one can easily show (e.g., see [64, p. 99]) that $p$ is a Hurewicz fibration (i.e., that $p$ has the homotopy lifting property for all spaces [64]). Now let $\theta: B \rightarrow C$ be a map and let $\pi: E_{\theta} \rightarrow B$ be the fiber space over $B$ induced by $\theta$ from $p$. Thus $E_{\theta}$ is the subspace of $B \times P C$ consisting of all pairs $(b, \lambda)$ such that $\theta(b)=\lambda(1)$. We call $\theta$ the classifying map for $\pi$.

Lemma 1. Let $X$ be a space and $f$ a map from $X$ to $B$. Then $f$ lifts to $E_{\theta}$ if, and only if, the map $\theta$ of is null-homotopic.

We rephrase the result slightly. For any pair of spaces $K$ and $L$ we denote by $[K, L]$ the homotopy classes of maps from $K$ to $L$. Given a third space $M$ and a map $f: K \rightarrow L$ we denote by

$$
f^{*}:[L, M] \rightarrow[K, M]
$$

the set map defined by

$$
f^{*}[\omega]=[\omega \circ f]
$$

where $\omega: L \rightarrow M$ and [. . ] denotes homotopy class. (In what follows it will simplify matters if we abuse the notation and let $\omega$ stand for both the map and the homotopy class: thus the above equation becomes

$$
f^{*} \omega=\omega \circ f \text {.) }
$$

In this notation Lemma 1 becomes:

$f$ lifts to $E_{\theta}$ if and only if $f^{*} \theta=0$, where 0 denotes the homotopy class of the constant map. 
We now come to the important idea of the Postnikov resolution. Suppose that $\pi: T \rightarrow B$ is any map, and suppose that we have a sequence of spaces and maps $\varepsilon=\left(E_{i}, p_{i}, q_{i}\right), i \geqq 1$, giving a commutative diagram, as shown below. (That is, we assume that $p_{i} \circ q_{i}=q_{i-1}$, for $i \geqq 2$, and $p_{1} \circ q_{1}=\pi$.)

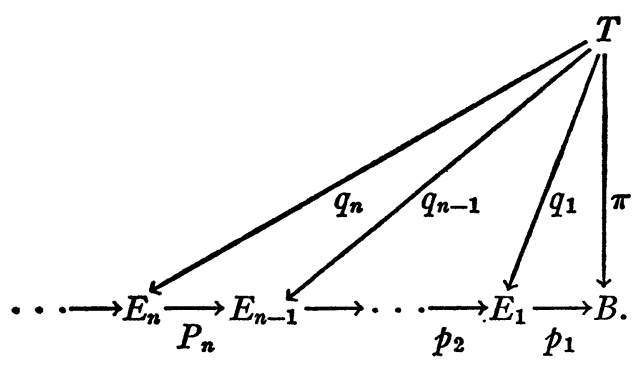

Definition 3. $\&$ is a Postnikov resolution of $\pi$ if the following conditions obtain:

(1) there is a sequence $\left\{r_{n}\right\}$ of integers with

$$
1 \leqq r_{1}<r_{2}<\cdots<r_{n}<\cdots,
$$

such that the morphism

$$
q_{r_{n}} *: \pi_{i}(T, *) \rightarrow \pi_{i}\left(E_{r_{n}}, *\right)
$$

is bijective for $0 \leqq i<n$ and surjective for $i=n$.

(2) For each $n \geqq 1$, there is a space $C_{n}$ and a map $\theta_{n}: E_{n-1} \rightarrow C_{n}$ such that the map $p_{n}: E_{n} \rightarrow E_{n-1}$ is the fibration with classifying map $\theta_{n}$. (We set $E_{0}=B$.)

From property (1) one easily deduces the following

Fact 7. Let $\varepsilon$ be a Postnikov resolution of $\pi$, as given above. Suppose that $X$ is a finite-dimensional complex and $f$ a map $X \rightarrow B$. Then $f$ lifts to $T$ if and only if $f$ lifts to $E_{r_{n}}$, where $n=\operatorname{dim} X$.

Thus we have reduced our problem to a finite succession of liftings, each of which can be studied by using Lemma 1.

Suppose that $f$ lifts to $E_{k-1}(k \geqq 1)$. Define

$$
\theta_{k}(f)=\bigcup_{0} g^{*} \theta_{k} \subset\left[X, C_{k}\right],
$$

where $g$ runs over all liftings of $f$ to $E_{k-1}$. By Lemma 1 we have

LEMMA 2. $f$ lifts to $E_{k}$ if and only if $f$ lifts to $E_{k-1}$ and $0 \in \theta_{k}(f)$.

In principle, then, the lifting problem is solved-assuming we have the resolution $\mathcal{E}$ and assuming we can compute the sets $\theta_{k}(f)$. 
We now discuss these two assumptions.

Existence of the Postnikov Resolution. Suppose that $\pi$ : $T \rightarrow B$ is a fibration such that $T$ and $B$ are $C W$-complexes. (Up to weak homotopy type, any map can be altered to satisfy these hypotheses; see $\$ \$ 2.8 .9$ and 7.8.1 in [64].) We assume moreover that the fiber of $\pi, F$, is 1-connected and that the fundamental group of $B$ acts trivally on the homotopy groups of $F$.

Theorem 31 (Postnikov, J. Moore). There exists a Postnikov resolution for $\pi$.

Postnikov proved this for the case $B$ is a point [54]; Moore then proved the general case, in the setting of semisimplicial complexes [46]. Hermann [20] gave the proof for an ordinary fibration, as did Eckmann-Hilton (see [22]). (The condition that $F$ is 1-connected can be somewhat relaxed; see the proof of the theorem given by Spanier $[64, \$ 8.3]$.

By the proof given for the theorem, each space $C_{r}$ is an EilenbergMacLane space $K\left(\pi_{r} F, r+1\right)$. Thus given a complex $X$ and a map $f: X \rightarrow E_{r-1}, \theta_{r}(f)$ is a collection of cohomology classes in $H^{r+1}\left(X ; \pi_{r} F\right)$. Thus for the Postnikov resolution to be a successful tool we must deal with the following problem.

Problem 15. Give a general method for computing the cohomology sets $\theta_{r}(f)$.

We indicate below a partial solution to this problem.

REMARK. Recently Mahowald [37], [15] has introduced several important modifications in the construction of a Postnikov resolution. In particular he shows that often one can take the spaces $C_{r}$ to be products of several Eilenberg-MacLane spaces. For an expository treatment of Postnikov resolutions, see $[72, \$ \$ 1-5]$.

Calculation of Postnikov invariants. We consider a schematic version of this problem, as shown in the following diagram. (In general we set $K_{s}=K\left(Z_{2}, s\right), s \geqq 1$.)

$(*)$

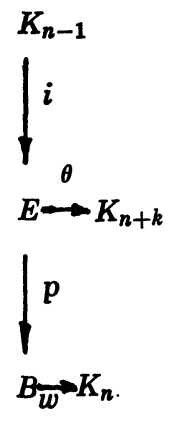


Here $\pi$ is an arbitrary map (between complexes) and $w$ is a class in $H^{n}(B)$ (mod 2 coefficients) such that $\pi^{*} w=0 . p$ is the fibration with $w$ as classifying map and $q$ is a lifting of $\pi$, so that $p \circ q=\pi . K_{n-1}$ $=\Omega K_{n}$ and $i$ denotes the fiber inclusion. Finally, $\theta$ is a class in $H^{n+k}(E)$ such that $q^{*} \theta=0$. Given a complex $X$ and a map $f: X \rightarrow B$ such that $f^{*} w=0$, we define

$$
\theta(f)=\bigcup_{0} g^{*} \theta
$$

where $g$ runs over all liftings of $f$ to $E$. Thus, $\theta(f) \subset H^{n+k}(X)$ and Problem 15 becomes

Problem 16. Compute the set $\theta(f)$.

We now make several simplifying assumptions.

(1) $0<k<$ connectivity of $B<n$.

(2) $\pi^{*} H^{n+k}(B)=H^{n+k}(T)(\bmod 2)$.

(3) Kernel $\pi^{*}=$ kernel $p^{*}$, in dimension $n+k$.

Let $\iota$ denote the fundamental class of $K_{n-1}, \iota \in H^{n-1}\left(K_{n-1}\right)$. By (1) there is an operation $\alpha$ in the mod 2 Steenrod algebra, $A_{2}$, such that (4) $i^{*} \theta=\alpha \iota$.

Definition 4. A class $V \in H^{*}(B)$ will be called a generating class for $\theta$ if the following conditions are satisfied: there exist operations $\beta, \gamma_{1}, \cdots, \gamma_{r}, \delta_{1}, \cdots, \delta_{r}$ in $A_{2}$ such that

(A) $w=\beta V, \delta_{i} V=0,1 \leqq i \leqq r$,

(B) $\alpha \beta+\sum_{i} \gamma_{i} \delta_{i}=0$.

REMARK. Relation (B) can be either an actual relation in the algebra $A_{2}$ (i.e., a "stable" relation); or it may simply be a relation among cohomology operations which holds on all cohomology classes with degree less than a given integer. We illustrate both possibilities in the examples given below.

Adams [1] has defined the notion of a secondary cohomology operation associated with a relation in $A_{2}$. Let $\Phi$ be an operation associated with (B). Then $\Phi$ is defined on those cohomology classes $u$ of a space $X$ such that $\beta u=0$ and $\delta_{i} u=0,1 \leqq i \leqq r$. Moreover, if we set

$$
\operatorname{Indet}(X ; \Phi)=\alpha H^{*}(X)+\sum_{i} \gamma_{i} H^{*}(X),
$$

then $\Phi(u) \in H^{*}(X) / \operatorname{Indet}^{*}(X ; \Phi)$. Notice that by (A), $\Phi$ is defined on $p^{*} V$ and $\pi^{*} V$. The following result is a special case of Theorem 5.9 in [74].

Theorem 32. Suppose that $\operatorname{Indet}^{n+k}(T ; \Phi)=\pi^{*} \operatorname{Indet}^{n+k}(B ; \Phi)$. Then for any class $m \in H^{n+k}(B)$ such that $\pi^{*} m \in \Phi\left(\pi^{*} V\right)$, we have 


$$
\theta+p^{*} m \in \Phi\left(p^{*} V\right)
$$

By naturality of secondary operations we obtain at once

Corollary 3. Let $X$ be a complex and $f: X \rightarrow B$ a map such that $f^{*} w=0$.

Suppose that

$$
\alpha H^{n-1}(X) \supset \sum_{i} \gamma_{i} H^{n+k-c_{i}}(X),
$$

where $c_{i}=$ degree $\gamma_{i}$. Then

$$
\theta(f)+\left\{f^{*} m\right\}=\Phi\left(f^{*} V\right)
$$

where $\{.$.$\} denotes the coset in H^{n+k}(X)$ with respect to the subgroup $\alpha H^{n-1}(X)$. In particular, if $0 \in \Phi\left(\pi^{*} V\right)$, then

$$
\theta(f)=\Phi\left(f^{*} V\right) \text {. }
$$

We give two examples to illustrate the corollary. Both deal with the question: when does an $n$-dimensional vector bundle $\xi$ have two independent cross-sections? If $\xi$ is over a complex $X$ then by using the classifying bundle we can regard $\xi$ as a map $X \rightarrow B_{n}$; and hence $\xi$ has two independent sections if and only if it lifts to $E_{n}(2)$. Now one can show that the space $E_{n}(2)$ has the same homotopy type as $B_{n-2}$, with the natural map $\pi: B_{n-2} \rightarrow B_{n}$ playing the role of the projection $E_{n}(2) \rightarrow B_{n}$. Thus we study the lifting of $\xi$ to $B_{n-2}$.

We now assume that $n$ is odd. The fiber of $\pi$ is the Stiefel manifold $V_{n, 2}$-a space which is $(n-3)$-connected, with $\pi_{n-2} V_{n, 2} \approx \pi_{n-1} V_{n, 2}=Z_{2}$ (see [65], [50]). A Postnikov resolution for $\pi$ can be constructed following Theorem 31 ; the first stage is given below:

$(* *)$

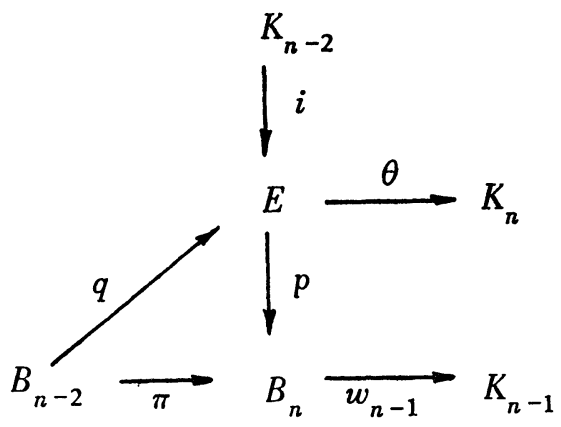

Here $w_{n-1}$ denotes the universal Stiefel-Whitney class in $H^{n-1}\left(B_{n}\right)$ (see $[72]$ ). 
LEMмA 3. The map $q$ and the class $\theta$ can be chosen so that

(1) $q^{*} \theta=0, i^{*} \theta=S q^{2} \iota$.

(2) If $X$ is a complex of dimension $\leqq n$ and $\xi: X \rightarrow B_{n}$ is a bundle such that $w_{n-1}(\xi)=0$, then $\xi$ has two independent sections if and only if

$$
0 \in \theta(\xi) \subset H^{n}(X) .
$$

Notice that if we restrict $\xi$ to $X^{n-1}$, then by (2) $\xi$ has two independent sections. Thus $\theta(\xi)$ represents the "index" to a 2 -field defined over $X^{n-1}$-i.e., if $X$ is a manifold $M$ and $\xi=\tau M$, then $\theta(\tau M)$ represents the index to a 2-field with finite singularities.

Depending on whether $n \equiv 1$ or $n \equiv 3 \bmod 4$, we give two methods of using Corollary 3 to compute $\theta(\xi)$.

EXAMPIE 1. $n \equiv 3 \bmod 4$.

In order to use Corollary 3 we must verify hypotheses (1)-(3). Now (2) and (3) are satisfied, while (1) is not. To overcome this problem we assume: in diagram (**) the spaces $B_{j}(j=n, n-2)$ are taken to be the classifying spaces for spin $j$-plane bundles-i.e., bundles $\xi$ such that $w_{1} \xi=w_{2} \xi=0 . B_{j}$ is then 3 -connected $(j \geqq 4)$ and so hypothesis (1) is satisfied.

Lemma 4. Suppose that $B_{n-2}$ and $B_{n}$, in diagram (**), denote classifying spaces for spin bundles. Assume that $n \equiv 3 \bmod 4, n>3$. Then $w_{n-3} \in H^{n-3}\left(B_{n}\right)$ is a generating class for $\theta$.

Proof. By Lemma 3, $\alpha=S q^{2}$. We take

$$
\beta=S q^{2}, \quad \gamma_{1}=S q^{1}, \quad \delta_{1}=S q^{2} S q^{1} .
$$

Then, (A) is satisfied because (setting $n=4 s+3$ ),

$$
S q^{2} W_{4 s}=W_{48+2}, \quad S q^{2} S q^{1} w_{4 s}=0 .
$$

(We use here the fact that $B_{n}$ is the classifying space for spin bundles.) And condition (B) is fulfilled, since one has the relation

(B') $S q^{2} S q^{2}+S q^{1}\left(S q^{2} S q^{1}\right)=0$, in $A_{2}$ [3].

Let $\Phi$ be a secondary operation associated with relation $\left(B^{\prime}\right)$. One can show that $0 \in \Phi\left(\pi^{*} w_{48}\right)$ and so we can take $m=0$ in Theorem 32 . Applying Corollary 3 we obtain

Theorem 33. Let $X$ be a complex of dimension $4 s+3$ and let $\xi$ be a $(4 s+3)$-plane bundle over $X$ such that

$$
w_{1} \xi=w_{2} \xi=w_{42+2} \xi=0 .
$$

Suppose that

$$
S q^{2} H^{4 s+1}(X) \supset S q^{1} H^{4 s+2}(X)
$$


Then $\theta(\xi)=\Phi\left(w_{48} \xi\right)$ in $H^{48+3}(X) / S q^{2} H^{4 s+1}(X)$. Thus $\xi$ has two independent sections if and only if $0 \in \Phi\left(w_{48} \xi\right)$.

We have computed $\theta(\xi)$ in terms of the operation $\Phi$. But this is a real gain, for one has several methods available for computing secondary operations. (A trivial example: if $w_{4 s} \xi=0$, then $\theta(\xi)=\Phi\left(w_{48} \xi\right.$ ) $=\Phi(0)=0$.) In particular, if $X$ is a spin manifold with $\xi$ its tangent bundle, then one can show that $0 \in \Phi\left(w_{4 s} \xi\right)$. This proves that a spin manifold of dimension $4 s+3$ has span $\geqq 2$.

REMARK. Hypothesis (1) made above is unduly restrictive. In fact the theory can be developed without it. One replaces the ordinary Steenrod operations with twisted cohomology operations. (See [16] and [42]; an exposition of these is given in [74, \$4].) Using these operations one can continue to define a generating class and prove the analogue of Theorem 32, without assuming hypothesis (1). By this method one establishes Theorem 2 in its full generality, without the assumption (made above) that $M$ is a spin manifold. For complete details, see [74].

EXAMPLE II. $n \equiv 1 \bmod 4$. Set $n=4 s+1, s \geqq 1$. The method given in Example I will no longer work, because $w_{48-2}$ is not a generating class for $\theta$. We describe here a second method.

With each $n$-plane bundle $\xi$ over a space $X$ one associates a new space $T \xi$, the Thom space of $\xi$. (Roughly speaking $T \xi$ is the "disk" bundle of $\xi$ with the "sphere" bundle collapsed to a point. See [30], [64] for details.) $\tilde{H}^{*}(T \xi)$ is a free module over $H^{*}(X)$ on one generator $U \in H^{n}(T X)$. ( $U$ is called the Thom class [30], [71].) We assume now that our bundles are oriented $n$-plane bundles; the class $U$ can now be taken as an integral cohomology class.

In diagram (**) we now take $B_{n-2}, B_{n}$ to be classifying spaces for oriented bundles. Let

$$
T=T\left(\gamma_{n}\right), \quad T^{\prime}=T\left(\pi^{*} \gamma_{n}\right) .
$$

The map $\pi$ induces a map $T \pi: T^{\prime} \rightarrow T$. Consider the following diagram:

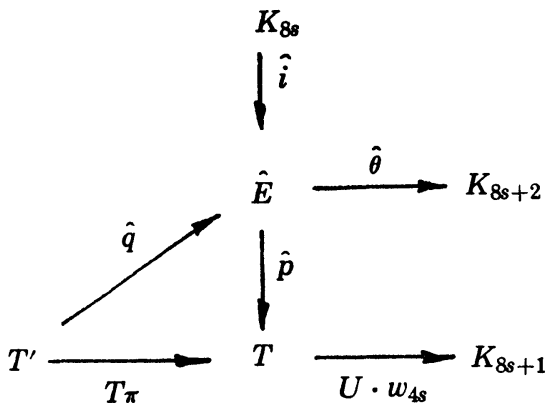


Here $\hat{p}$ is the induced fibration with $U \cdot w_{4 s}$ as classifying map. Since $\pi^{*} w_{4 \mathrm{~s}}=0$, one has that $(T \pi)^{*}\left(U \cdot w_{48}\right)=0$, and so $T \pi$ lifts to a map $\hat{q} ; \hat{\imath}$ is the fiber inclusion and $\hat{\theta}$ is a class in $H^{8 s+2}(\hat{E})$.

Let $\xi: X \rightarrow B_{48+1}$ be an oriented bundle and let $t: T \xi \rightarrow T$ be the induced map on Thom spaces.

Lemma 5. There is a class $\hat{\theta}$ in $H^{8 s+2}(\hat{E})$ (defined independently of $X$ ) so that

(1) $\hat{\imath} * \hat{\theta}=S q^{2} \iota, \hat{q}^{*} \hat{\theta}^{*}=0$.

(2) Suppose that $w_{48} \xi=0$, so that $\theta(\xi)$ is defined. Then, $t$ lifts to $\hat{E}$ and $\hat{\theta}(t)=U_{X} \cdot \theta(\xi)$, where $U_{X}$ denotes the Thom class of $\xi$.

Since multiplication by $U_{X}$ gives an isomorphism in cohomology, to compute $\theta(\xi)$, it suffices to compute $U_{X} \cdot \theta(\xi)$, i.e., $\hat{\theta}(t)$.

(The idea of "lifting the Postnikov invariant to the Thom space" is given by Mahowald-Peterson in [39]. For a general treatment of the matter, see $[76, \S 6]$.)

Lemma 6. $U$ is a generating class for $\hat{\theta}$.

Proof. Now $\hat{p}$ is induced by the class $U \cdot w_{48}$. But by Thom [71],

$$
U \cdot w_{4 s}=S q^{4 s} U
$$

thus, in (A) above, we take $\beta=S q^{4 s}$. By Lemma 5, $\alpha=S q^{2}$. But by the Adem relations [3], one has

$$
S q^{2} S q^{4 s}=0,
$$

on integral cohomology classes of degree $\leqq 4 s+1$. Thus relation $\left(^{*}\right)$ satisfies hypothesis (B), and so $U$ is a generating class as claimed.

Let $\Omega$ be a secondary cohomology operation [1] associated with relation $\left(^{*}\right)$. One can show that $\Omega$ can be chosen so that

$$
(T \pi)^{*}\left(U \cdot w_{2} w_{4 s+1}\right) \in \Omega\left(T \pi^{*} U\right)
$$

(see [39]). Thus we can take $m=w_{2} w_{4 s-1}$ in Theorem 32. As a result we obtain

Corollary 4. Let $X$ be a complex and $\xi$ an oriented $(4 s+1)$-plane bundle over $X$ such that $w_{48} \xi=0$ and $S q^{2} H^{s s}(T(\xi))=0$. Then

$$
U_{X}\left(\theta \cdot(\xi)+w_{2}(\xi) \cdot w_{48-1}(\xi)\right)=\Omega\left(U_{X}\right),
$$

where $U_{X}$ denotes the Thom class of $\xi$.

To utilize the corollary, we must calculate the operation $\Omega$ on $U_{X}$. This has been done in two different situations. 
(1) Suppose that $X$ is a compact orientable manifold $M$, and that $\xi$ is the normal bundle to an immersion of $M$ in $R^{8 s+2}$. Then, $\Omega\left(U_{X}\right)=0$ and so we obtain

$$
\theta(\xi)=w_{2}(\xi) \cdot w_{4 s-1}(\xi) .
$$

This type of calculation has been exploited by Mahowald-Peterson [39] to obtain immersion theorems for manifolds, using the theorem of Hirsch [23].

(2) Suppose that $M$ is a compact spin manifold and that $\xi$ is the tangent bundle of $M$. By definition, $w_{2} M\left(=w_{2} \xi\right)=0$, and so Corollary 4 reads $U_{M}\left(\theta\left(\tau_{M}\right)\right)=\Omega\left(U_{M}\right)$. One can show (see [76])

THEOREM 34. $\Omega\left(U_{M}\right)=U_{M}\left(\hat{\chi}_{2} M \cdot \mu\right)$ where $\mu$ is the generator of $H^{4 s+1}\left(M ; Z_{2}\right)$.

Therefore, we obtain $\theta\left(\tau_{M}\right)=\hat{\chi}_{2} M \cdot \mu$, and so $\hat{\chi}_{2} M$ is the index of a 2 -field on $M$, as given in Theorem 3 . For a detailed discussion of this method, see [76] and [80].

6. Corrigenda. This is an appropriate place to correct errors in my previous papers.

(i) On p. 86 of [76], it is not correct to say that the index "... is independent of the orientation of $M^{\prime \prime}$ (see also p. 148 of [80]). In particular, Theorem 5 in this paper is an example of the dependence of the index on the choice of orientation. It is true, however, that the theorems given in [76] and [77] are independent of the orientation.

(ii) Theorem 6.7 of [81] is incomplete for the case $r=4$. The error lies in the fact that $\pi_{4 s+2}\left(V_{4 s+3,4}\right)$ is $Z_{2} \oplus Z_{2}$, rather than $Z_{2}$. As a result, an additional obstruction occurs in dimension $n$. However, this can be evaluated by a tertiary operation exactly as in Theorem 4.6 of [75]. In particular, this obstruction is zero for the tangent bundles of the manifolds considered in Theorem 1.2 [81], and so Theorem 1.2 remains true. The method of proof is that of Theorem 1.3 in [75].

(iii) Because of the use made of the Serre isomorphism theorem in [72], the hypotheses given on p. 12 of [72] (first sentence) should be strengthened to include: $\mathrm{E}$ and $\mathrm{B}$ are 1-connected.

\section{BIBLIOGRAPHY}

1. F. Adams, On the non-existence of elements of Hopf invariant one, Ann. of Math. (2) 72 (1960), 20-104.

2. - Vector fields on spheres, Ann. of Math. (2) 75 (1962), 603-632.

3. J. Adem, "The relations on Steenrod powers of cohomology classes" in Algebraic geometry and topology, Princeton Univ. Press, Princeton, N. J., 1967, pp. 191238. 
4. M. Atiyah and F. Hirzebruch, Vector bundles and homogeneous spaces, Proc. Sympos. Pure Math., vol. 3, Amer. Math. Soc., Providence, R.I., 1961, pp. 7-38.

5. M. Atiyah and I. Singer, The index of elliptic operators. III, Ann. of Math. (2) 87 (1968), 546-604.

6. M. Barratt and M. Mahowald, The metastable homotopy of $O(n)$, Bull. Amer. Math. Soc. 70 (1964), 758-760.

7. A. Borel and F. Hirzebruch, Characteristic classes and homogenous spaces. I, II, III, Amer. J. Math. 80 (1958), 458-538; 81 (1959), 315-382; 82 (1960), 491-504.

8. R. Bott, The stable homotopy of the classical groups, Ann. of Math. (2) 70 (1959), 313-337.

9. G. Bredon, Sheaf theory, McGraw-Hill, New York, 1967.

10. G. Bredon and A. Kosinski, Vector fields on r-manifolds, Ann. of Math. (2) 84 (1966), 85-90.

11. B. Eckmann, Systeme von Richtungsfeldern in Sphären und stetige Lösungen komplexer linearer Gleichungen, Comment. Math. Helv. 15 (1943), 1-26.

12. - Gruppentheoretischer Beweis des Satzes von Hurwitz-Radon über die Komposition quadratischer Formen, Comment. Math. Helv. 15 (1943), 358-366.

13. D. Frank (to appear).

14. D. Frank and E. Thomas, $A$ generalization of the Steenrod-Whitehead theorem, Topology 7 (1968), 311-316.

15. S. Gitler and M. Mahowald, The geometric dimension of real stable vector bundles, Bol. Soc. Mat. Mexicana 11 (1966), 85-107. 266.

16. S. Gitler and J. Stasheff, The first exotic class of BF, Topology 4 (1965), 257-

17. A. Haefliger, Variêtes feuilletêes, Ann. Scuola Norm. Sup. Pisa (3) 16 (1962), 367-397.

18. - Structure feuilletées et cohomologie d valeur dans un faisceau de groupoides, Comment Math. Helv. 32 (1958), 248-329.

19. A. Haefliger and M. Hirsch, Immersions in the stable range, Ann. of Math. (2) 75 (1962), 231-241.

20. R. Hermann, Secondary obstructions for fiber spaces, Bull. Amer. Math. Soc. 65 (1959), 5-8.

21. - Obstruction theory for fiber spaces, Illinois J. Math. 4 (1960), 9-27.

22. P. Hilton, Homotopy theory and duality, Gordon and Breach, New York, 1966.

23. M. Hirsch, Immersions of manifolds, Trans. Amer. Math. Soc. 93 (1959), 242-276.

24. F. Hirzebruch, Neue topologische Methoden in der algebraischen Geometrie, Ergebnisse der Math. und Grenzdebiete, Heft 9, Springer-Verlag, Berlin, 1956; 1962; English transl., Die Grundlehren der Wissenschaften, Band 131, Springer-Verlag, New York, 1966.

25. F. Hirzebruch and K. H. Mayer, $O(n)$-mannigfaltigkeiten, exotische sphären und singularitäten, Lecturen Notes in Mathematics No. 57, Springer-Verlag, Berlin, 1968.

26. F. Hirzebruch and H. Hopf, Felder von flächenelementen in 4-dimensionalen mannigfaltigkeiten, Math. Ann. 136 (1958), 156-172.

27. H. Hopf, Vectorfelder in n-dimensionalen mannigfaltigkeiten, Math. Ann. 96 (1927), 225-260.

28. A. Hurwitz, Mathematische Werke. Funktionentheorie. Band I, Birkhäuser Verlag, Basel, 1962, pp. 565-571. 
29. - Mathematische Werke. Zahlentheorie, Algebra und Geometrie. Band II, Birkhäuser Verlag, Basel, 1963, pp. 641-666.

30. D. Husemoller, Fibre bundles, McGraw-Hill, New York, 1966.

31. M. Kervaire, Courbure integrale generalisée et homotopie, Math. Ann. 131 (1956), 219-252.

32. - Non-parallelizability of the $n$-sphere for $n>7$, Proc. Nat. Acad. Sci. U.S.A. 44 (1958), 280-283.

33. M. Kervaire and J. Milnor, Groups of homotopy spheres. I, Ann. of Math. (2) 77 (1963), 504-537.

34. S. Lang, Introduction to differentiable manifolds, Interscience, New York, 1962.

35. W. B. R. Lickorish, A foliation for 3-manifolds, Ann. of Math. (2) 92 (1965), 414-420.

36. E. Lima, Commuting vector fields on $S^{3}$, Ann. of Math. (2) 81 (1965), 70-81.

37. M. Mahowald, On obstruction theory in orientable fiber bundles, Trans. Amer. Math. Soc. 110 (1964), 315-349.

38. - On the metastable homotopy of $O(n)$, Proc. Amer. Math. Soc. 19 (1968), 639-641.

39. M. Mahowald and F. Peterson, Secondary cohomology operations on the Thom class, Topology 2 (1964), 367-377.

40. W. Massey, On the Stiefel-Whitney classes of a manifold, Amer. J. Math. 82 (1960), 92-102.

41. - On the Stiefel-Whitney classes of a manifold. II, Proc. Amer. Math. Soc. 13 (1962), 938-942.

41a. K. H. Mayer, Elliptische Differentialoperatoren und Ganzzahligkeitssätze für charakterische Zahlen, Topology 4 (1965), 295-313.

42. J. F. McClendon, Higher order twisted cohomology operations, Thesis, University of California, Berkeley, Calif., 1966.

43. J. Milnor, Lectures on characteristic classes, Mimeographed Notes, Princeton University, Princeton, N. J., 1957.

44. - Some consequences of a theorem of Bott, Ann. of Math. (2) 68 (1958), 444-449.

45. - Spin structures on manifolds, Enseignement Math. (2) 9 (1963), 198-203.

46. J. C. Moore, Semi-simplicial complexes and Postnikov systems, Internat. Sympos. on Algebraic Topology, Universidad Nacional Autónoma de México and UNESCO, Mexico City, 1958, pp. 232-247.

47. S. P. Novikov, The topology of foliations, Trudy Moscov. Mat. Obšč. 14 (1965), 248-278 = Trans. Moscow Math. Soc. 1965, 268-304.

48. - The topology summer institute, Uspehi Mat. Nauk 20 (1965) = Russian Math. Surveys 20 (1965), 145-168.

49. P. Olum, Mappings of manifolds and the notion of degree, Ann. of Math. (2) 58 (1953), 458-480.

50. G. Paechter, The groups $\pi_{r}\left(V_{n, m}\right)$. I, Quart. J. Math. Oxford Ser. (2) 7 (1956), 249-268.

51. R. Palais, $A$ global formulation of the Lie theory of transformation groups, Mem. Amer. Math. Soc. No. 22 (1957).

52. A. Phillips, Submersions of open manifolds, Topology 6 (1967), 171-206.

53. - Foliations on open manifolds. I, Comment. Math. Helv. 43 (1968), 204-211. 
54. M. Postnikov, Investigations in the homotopy theory of continuous mappings. I, II, Trudy Mat. Inst. Steklov. 46 (1955); English transl., Amer. Math. Soc. Transl. (2) 7 (1957), 1-98, 98-134.

55. J. Radon, Lineare scharen orthogonaler matrizen, Abh. Math. Sem. Univ. Hamburg 1 (1923), 1-14.

56. G. Reeb, Sur certaines propriétés topologiques des variêtés feuilletées, Actualitiés Sci. Indust. No. 1183 = Publ. Inst. Math. Univ. Strasbourg, Hermann, Paris, 1952.

57. B. Reinhart, Characteristic numbers of foliated manifolds, Topology 6 (1967), 467-472.

58. H. Rosenberg, Foliations by planes, Topology 7 (1968), 131-138.

59. R. Sacksteder, Foliations and pseudogroups, Amer. J. Math. 87 (1965), 79-102.

60. - Degeneracy of orbits of actions of $R^{m}$ on a manifold, Comment. Math. Helv. 41 (1966), 1-9.

61. H. Samelson, $A$ note on the Bockstein operator, Proc. Amer. Math. Soc. 15 (1964), 450-453.

62. B. M. Sanderson, Immersions and embeddings of projective spaces, Proc. London Math. Soc. (3) 14 (1964), 137-153.

63. J.-P. Serre, Homologie singulière des espaces fibrés, Ann. of Math. (2) 54 (1951), 425-505.

64. E. Spanier, Algebraic topology, McGraw-Hill, New York, 1966.

65. N. Steenrod, The topology of fiber bundles, Princeton Univ. Press, Princeton, N. J., 1951.

66. N. Steenrod and J. H. C. Whitehead, Vector fields on the n-sphere, Proc. Nat. Acad. Sci. U.S.A. 37 (1957), 58-63.

67. S. Sternberg, Lectures on differential geometry, Prentice-Hall, Englewood Cliffs, N. J., 1964.

68. E. Stiefel, Richtungsfelder und Fernparallelismus in n-dimensionalen Mannigfaltigkeiten, Comment. Math. Helv. 8 (1936), 305-353.

69. R. Strong, Determination of $H^{*}(B O(k, \cdots, \infty))$ and $H^{*}\left(B O(k, \cdots, \infty), Z_{2}\right)$, Trans. Amer. Math. Soc. 107 (1963), 526-544.

70. W. Sutherland, Fiber homotopy equivalence and vector fields, Proc. London Math. Soc. 15 (1965), 543-556.

71. R. Thom, Espaces fibrés en sphères et carrés de Steenrod, Ann. Sci. Ècole Norm. Sup. (3) 69 (1952), 109-182.

72. E. Thomas, Seminar on fiber spaces, Lecture Notes in Mathematics No. 13, Springer-Verlag, New York, 1966.

73. - Cross-sections of stably equivalent vector bundles, Quart. J. Math. Oxford Ser. (2) 17 (1966), 53-57.

74. - Postnikov invariants and higher order cohomology operations, Ann. of Math. (2) 85 (1967), 184-217.

75. - Real and complex vector fields on manifolds, J. Math. Mech. 16 (1967), 1183-1206. 110.

76. — The index of a tangent 2-field, Comment. Math. Helv. 42 (1967), 86-

77. - Fields of tangent 2-planes on even-dimensional manifolds, Ann. of Math. (2) 86 (1967), 349-361.

78. — , Fields of tangent $k$-planes on manifolds, Invent. Math. 3 (1967), 334347.

79. —, Vector fields on low dimensional manifolds, Math. Z. 103 (1968), 85-93. 
80. - "Characteristic classes and differentiable manifolds" in Classi Caratteristiche e questioni connesse, Edizioni Cremonese, Rome, 1967, pp. 115-187.

81. —, The span of a manifold, Quart. J. Math. Oxford Ser. 19 (1968), 225244.

82. G. W. Whitehead, Homotopy properties of the real orthogonal groups, Ann. of Math. (2) 43 (1942), 132-146.

83. - On families of continuous vector fields over spheres, Ann. of Math. (2) 47 (1946), 779-785; correction to: 48 (1947), 782.

84. J. Wood, Foliations on 3-manifolds, Ann. of Math. (to appear).

85. W. T. Wu, Les i-carrés dans une variété grassmannienne, C. R. Acad. Sci. Paris 230 (1950), 918-920.

86. - Classes caractéristiques et i-carrés d'une variêté, C. R. Acad. Sci. Paris 230 (1950), 508-511.

University of California, Berkeley, California 94720 